\title{
The existence of steady flow in a collapsed tube
}

\author{
By O. E. JENSEN AND T. J. PEDLEY \\ Department of Applied Mathematics and Theoretical Physics, University of Cambridge, \\ Silver Street, Cambridge CB3 9EW, UK
}

(Received 28 July 1988 and in revised form 10 March 1989)

Self-excited oscillations arise during flow through a pressurized segment of collapsible tube, for a range of values of the time-independent controlling pressures. They come about either because there is an (unstable) steady flow corresponding to these pressures, or because no steady flow exists. We investigate the existence of steady flow in a one-dimensional collapsible-tube model, which takes account of both longitudinal tension and jet energy loss $E$ downstream of the narrowest point. For a given tube, the governing parameters are flow-rate $Q$, and transmural pressure $P$ at the downstream end of the collapsible segment. If $E=0$, there exists a range of $(Q, P)$-values for which no solutions exist; when $E \neq 0$ a solution is always found. For the case $E \neq 0$, predictions are made of pressure drop along the collapsible tube; these solutions are compared with experiment.

\section{Introduction}

Elastic tubes can collapse if the external pressure is high enough compared with the internal, and when a tube is collapsing it becomes so compliant that hydrodynamic pressure changes associated with flow through it can be large enough to influence the collapse process strongly. Such coupling of fluid flow through an elastic tube with collapse of the tube arises in a number of physiological and mechanical contexts (Shapiro $1977 a$ ). Laboratory experiments designed to examine the detailed mechanics or steady behaviour of slowly varying flow through finite lengths of collapsible tube, supported at each end (figure 1), are almost invariably complicated by the development of self-excited oscillations in cross-sectional area, outflow rate and pressure drop, and the investigation of such oscillations has led to a variety of different theoretical models and assertions concerning the physical mechanisms involved (see Cancelli \& Pedley 1985 for a brief survey and a long, but by no means complete, list of pertinent references). One difficulty is that there are several possible mechanisms, any one, or more than one, of which may be important in a given experiment, and very few of the early experimentalists recorded enough of the relevant quantitative details for a critical test of any particular model to be made. In the last few years, however, a new series of very careful experiments has been performed by Bertram $(1982,1986,1987)$ who, with all the different models in mind, has endeavoured to measure every one of the potentially relevant parameters. Furthermore, in the course of these experiments, he has discovered a wide variety of oscillatory behaviour in a fairly limited region of parameter space, and has been able to map out many of the transitions between them (see Bertram, Raymond \& Pedley 1989). This suggests that the finite, externally pressurized collapsible tube with fluid flow through it should be thought of as a dynamical system with a rich bifurcation structure, and provides a strong incentive to develop a good theoretical description of it. 


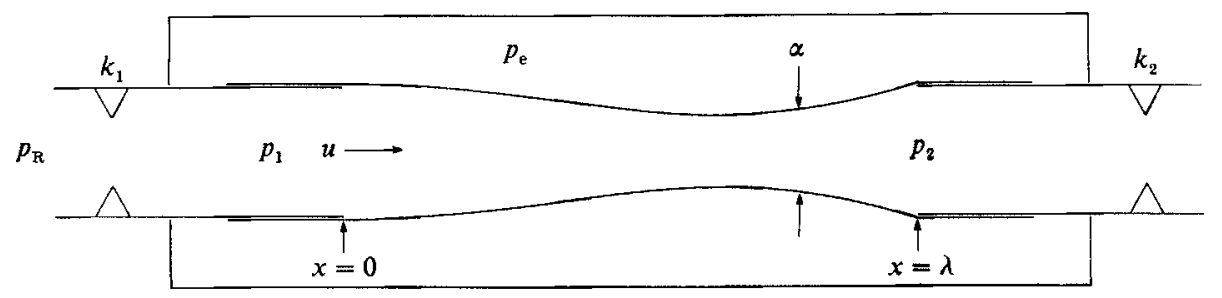

Figure 1. Conventional laboratory apparatus: an elastic tube is mounted at each end on two rigid tubes and contained in a pressurised chamber; $k_{1}$ and $k_{2}$ represent flow resistances.

Most previous models have been either lumped-parameter models, in which the geometry of the collapsible segment is represented by one or more time-dependent variables such as the cross-sectional area at the narrowest point, and the rigid parts of the system up- and downstream of the collapsible segment control both the existence and the form of the oscillations (e.g. Bertram \& Pedley 1982), or distributed, one-dimensional models in which the fact that the elastic tube can support propagating pressure waves is paramount, but the up- and downstream parts of the system are often ignored (e.g. Oates 1975). Lumped-parameter models are described by systems of nonlinear ordinary differential equations, but the distributed model requires a partial differential equation, hyperbolic in the simplest version. Experimental evidence suggests that unsteady behaviour sets in when the flow becomes critical, i.e. when the fluid speed becomes equal to the propagation speed of long waves somewhere in the tube (Brower \& Scholten 1975; Bonis \& Ribreau 1978), which implies that a lumped-parameter model cannot be adequate. On the other hand, the oscillations are affected by changes in the resistance of the system downstream, for example (Conrad 1969), so the collapsible tube cannot be considered in isolation. Cancelli \& Pedley (1985) proposed a hybrid model, of a finite length of collapsible tube mounted on rigid tubes at each end, but using the full onedimensional equations in the collapsible segment. A novel aspect of the model was the incorporation of two features previously identified as important but not commonly included: longitudinal tension in the tube wall, and energy loss in the separated flow downstream of the narrowest point. Indeed, these authors identified hysteresis in the unsteady separation process as an important factor in the development of oscillations.

All the results of Cancelli \& Pedley (1985) were obtained numerically, and a not very wide region of parameter space was studied. The desire to understand the collapsible tube as a dynamical system demands a more mathematical treatment. In this paper we make a start by analysing steady solutions of the model equations used by Cancelli \& Pedley (with a slight further simplification-see $\$ \S 2.2$ and 2.3), covering the whole of parameter space. These steady solutions are fixed points in the space of solutions to the full, time-dependent problem. At a given point in parameter space, unsteady behaviour can arise in one of two ways: $(a)$ if no steady solution exists, and $(b)$ if an existing steady solution is unstable. Here we shall concentrate exclusively on $(a)$.

Our analysis closely follows the approach used by Reyn (1987), who also examined the existence and uniqueness of steady flow through finite lengths of collapsible tube held open at the ends. He, too, incorporated longitudinal tension into his model, but he totally neglected all (viscous) energy loss in the system, while we include the jet loss downstream of the narrowest point. Also Reyn's description of the elastic 
properties of the tube was much more complicated than that used here, so his analysis was inevitably more cumbersome. The equations of our model are presented in $\S 2$, and in $\$ 3$ we analyse the loss-free case, and we show that the simplification in elastic properties makes no qualitative difference to Reyn's results. In $\$ 4$ the complete model is solved, and the presence of energy loss is shown to have important qualitative effects; in particular, there exists at least one steady solution at all points of parameter space at which the tube is collapsed (i.e. with minimum area less than that of the up- and downstream rigid tubes), whereas there are regions in which no solution exists when energy losses are zero. Finally, in $\$ 5$, we attempt a quantitative comparison of the theoretical predictions, in the form of graphs of pressure drop along the collapsible segment against flow rate, with three different sets of experiments: those of Conrad (1969), Bonis \& Ribreau (1978, 1981) and Bertram $(1986,1987)$. That pressure drop is of course zero in the loss-free case.

\section{The model}

The steady one-dimensional model developed below is based on that developed by Cancelli \& Pedley (1985). The quantities $\tilde{A}$ (tube cross-sectional area), $\bar{u}$ (crosssectionally averaged fluid velocity) and $\tilde{p}$ (internal pressure) are taken to be timeindependent functions of the longitudinal coordinate $\tilde{x}$. It is assumed that there are no transverse variations in $u$ or $\tilde{p}$, (which is more accurate for the time mean of turbulent flow than for laminar flow), except where flow separation takes place. The fluid is assumed incompressible, and has density $\rho$.

\subsection{Flow equations}

Since mass is conserved, the volume flux

is independent of $\tilde{x}$.

$$
\tilde{Q}=\tilde{A} \bar{u}
$$

The form of the equation of motion will depend on the mechanisms of energy loss. There are two such mechanisms in the flow through a collapsible tube, arising either from frictional forces between the fluid and the tube wall or from viscous dissipation in the region of separated flow downstream of a constriction in the tube. As Cancelli \& Pedley noted, the effect of friction is generally negligible in comparison to that of flow separation (the exceptional cases are when the tube is severely constricted along its entire length or when it is dilated along its length and no separation occurs), and we shall therefore neglect friction in our model.

As $\tilde{A}$ increases beyond a constriction in the tube, fluid velocity must decrease and so there is a rise in pressure. This adverse pressure gradient, if it is sufficiently large, leads to boundary-layer separation from the tube wall. At high flow rates a turbulent jet is formed and the flow may remain separated as far as the downstream end of the collapsed tube. In a case where there is no separation, there will be no energy dissipation and the equation of motion downstream of the constriction will be the same as that upstream, i.e.

$$
\bar{u} \bar{u}_{\tilde{x}}=-\frac{1}{\rho} \tilde{p}_{\tilde{x}}
$$

If, when there is separation, the jet remains parallel-sided there will be no pressure recovery as the mean velocity decreases, so the equation of motion becomes

$$
\tilde{p}_{\tilde{x}}=0 \text {. }
$$


Downstream of a constriction in practice there is a widening of the jet accompanied by some pressure recovery. This suggests that the equation can be written in the form

$$
\chi^{\bar{u}} \bar{u}_{\tilde{x}}=-\frac{1}{\rho} \tilde{p}_{\tilde{x}}
$$

where $0<\chi<1$. We follow Cancelli \& Pedley (1985) in taking $\chi$ to be a constant (compare the Borda-Carnot condition for overall pressure recovery downstream of an orifice, equivalent to $\chi=0.18$ for a $90 \%$ constriction); the precise value of $\chi$ turns out to be unimportant. The point of separation should be determined as the place where the adverse pressure gradient exceeds a positive critical value, a little way downstream of the point where the cross-sectional area is smallest. For convenience we assume that separation occurs at the point of smallest area, consistent with the model of Smith \& Duck (1980) for internal separated flow at large Reynolds number. Thus the equation of motion is taken to be (2.2) (i.e. (2.4) with $\chi=1$ ) upstream of the narrowest point, and downstream of it when there is no separation, and (2.4) with $0<\chi<1$ downstream when there is separation.

\subsection{The tube law}

The tube law is a relationship between transmural pressure and the local crosssectional area. In the absence of longitudinal tension or bending it is taken to be of the form

$$
\tilde{p}-\tilde{p}_{\mathrm{e}}=\tilde{P}(\tilde{A}),
$$

where $\tilde{p}_{\mathrm{e}}$ is the external pressure and

$$
\tilde{P}(\tilde{A})=K_{p} \mathscr{P}(\alpha)
$$

$K_{p}$ is a constant proportional to the circumferential bending stiffness of the tube wall (Shapiro $1977 b$ ). With $\tilde{A}_{0}$ the unstretched (circular) area and $k$ a constant, the following modified similarity law is used (again following Shapiro 1977b):

$$
\mathscr{P}(\alpha)= \begin{cases}1-\alpha^{-\frac{3}{2}} & \text { for } \alpha \equiv \tilde{A} / \tilde{A}_{0} \leqslant 1 \\ k(\alpha-1) & \text { for } \alpha \equiv \tilde{A} / \tilde{A}_{0}>1\end{cases}
$$

see figure 2 . The speed $\tilde{c}$ of propagation of small-amplitude waves when there is no mean flow is given by

$$
\tilde{c}^{2}=\frac{\tilde{A}}{\rho} \tilde{P}^{\prime}(\tilde{A}) \text {. }
$$

\subsection{Longitudinal tension}

Local collapse of the tube is accompanied by longitudinal as well as transverse bending of the wall. If the tube is stretched longitudinally, as in most of the experiments, then the longitudinal tension will oppose this bending and will contribute to the transmural pressure. Following MoClurken et al. (1981), we recognize that the cross-section of the tube is very flattened when it is collapsed, and therefore approximate the tube as a pair of two-dimensional membranes whose spacing is proportional to the local cross-sectional area. The tube law is accordingly modified as

$$
\tilde{p}-\tilde{p}_{\mathbf{e}}=\tilde{P}(\tilde{A})-\frac{T}{R}
$$

where $T$ is the longitudinal tension per unit perimeter and $R$ is the longitudinal radius of curvature. The expression for $R$ used by Cancelli \& Pedley (1985) contains 


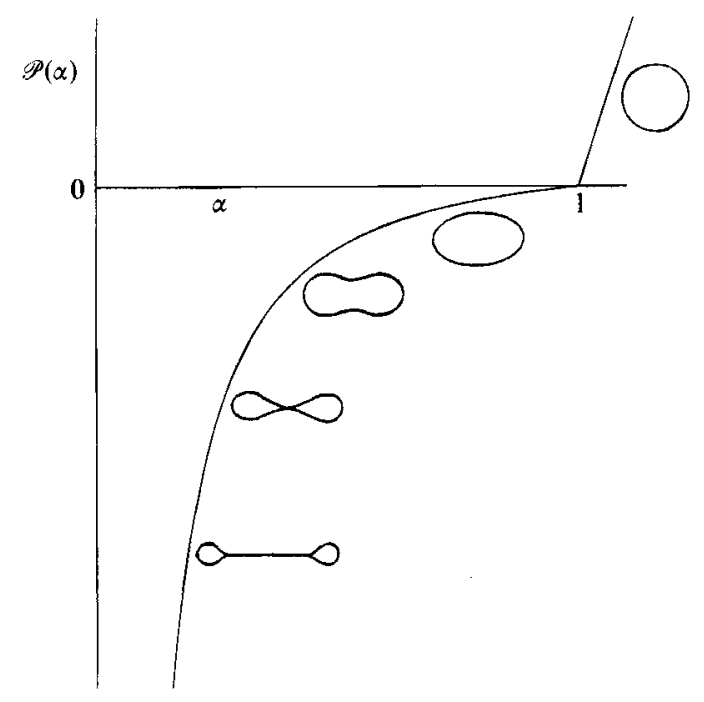

Figure 2. The tube law: non-dimensionalized transmural pressure is plotted against the nondimensionalized cross-sectional area $\alpha$ of a uniform elastic tube at zero longitudinal tension; the shape of the cross-section is shown for varying $\alpha$.

nonlinear terms which we neglect on the assumption that the tube area varies slowly with $\tilde{x}$, i.e. that

$$
\frac{D_{0} \tilde{A_{\tilde{x}}}}{\tilde{A_{0}}} \ll 1,
$$

where $D_{0}$ is the diameter of the unstretched tube, so in this paper we take

$$
\frac{1}{R}=\frac{D_{0} \tilde{A_{x} \tilde{x}}}{2 \tilde{A_{0}}} .
$$

The assumption will be inaccurate where there is a large change in tube area over a short axial distance, for example when the constriction is very close to the junction with the downstream rigid tube. It is hoped, however, that the influence of this term is much less important than that of those nonlinearities included in the analysis. Reyn (1987) used a more accurate description of tube wall elasticity, which added considerable complication to the mathematical analysis, but did not lead to qualitative differences in the results.

McClurken et al. (1981) also discussed the effect of longitudinal bending stiffness on transmural pressure, but concluded that tension normally plays a more significant role. We therefore neglect the bending stiffness; we assume also that any variations in $T$ with $\tilde{x}$ may be neglected: this is reasonable provided changes in $T$ with $\tilde{x}$, brought about by longitudinal skin friction for example, are much smaller than $T$ itself; this is consistent with the neglect of friction.

\subsection{Boundary conditions}

There are four boundary conditions to be applied to the fourth-order system formed by the above equations. $\tilde{x}=0$ and $\tilde{x}=L$ are the positions of the junctions of the segment of collapsible tube and the rigid tubes up- and downstream. Each rigid tube is assumed to have cross-sectional area $\tilde{A_{0}}$. Then two of the boundary conditions are

$$
\tilde{A}(0)=\tilde{A}(L)=\tilde{A_{0}},
$$


and the other two specify the upstream and downstream pressures:

$$
\begin{gathered}
\tilde{p}(0)=\tilde{p}_{\mathrm{R}}-\frac{1}{2} \rho \bar{u}^{2}(0)-k_{1} \tilde{A}_{0}^{2} \bar{u}^{2}(0), \\
\tilde{p}(L)=k_{2} \tilde{A}_{0}^{2} \bar{u}^{2}(L),
\end{gathered}
$$

where $k_{1}$ and $k_{2}$ are constants representing the properties of the constrictive valves upstream and downstream respectively. The downstream rigid tube is open to the atmosphere, and so (2.14) represents the pressure drop across the downstream constrictive valve (see figure 1). Far upstream, water flows from a static reservoir at pressure $\tilde{p}_{\mathbf{R}}$ into a rigid tube, down which there is a pressure drop due to the gain in momentum of the fluid and a further drop across the upstream valve, so that $\tilde{p}(0)$ is given by (2.13).

\subsection{Non-dimensionalization}

The following scales are used for non-dimensionalization : area $\tilde{A_{0}}$; pressure $K_{p}$ and velocity $c_{0}=\left(K_{p} / \rho\right)^{\frac{1}{2}}$. The appropriate lengthscale for longitudinal variation turns out to be

$$
\tilde{L}=\left(\frac{\tilde{T}}{\pi K_{p}}\right)^{\frac{1}{2}}
$$

where $\tilde{T}$ is the total longitudinal tension in the tube $\left(=\pi D_{0} T\right) ;\left(\tilde{L} / D_{0}\right)^{2}$ is proportional to the ratio of longitudinal stress to hoop stress in the tube when it is beginning to collapse, or equivalently to the longitudinal radius of curvature divided by the diameter, and ought to be large for the one-dimensional-flow assumption to be accurate. Let $x=\tilde{x} / \tilde{L}, \alpha=\tilde{A} / \tilde{A_{0}}, p=\tilde{p} / K_{p}$, and $u=\bar{u} / c_{0}$. Equation (2.8) for the wave speed $\tilde{c}$ of long waves becomes $c^{2}=\alpha \mathscr{P}^{\prime}(\alpha)$, but it should be remembered that longitudinal tension makes the system dispersive, so that there is no unique wave propagation speed.

The governing equations become

$$
\begin{gathered}
(u \alpha)_{x}=0, \\
\chi u u_{x}=-p_{x}, \\
p-p_{\mathrm{e}}=\mathscr{P}(\alpha)-\frac{1}{2} \alpha_{x x},
\end{gathered}
$$

where $\chi=1$ when the flow is attached. With $\lambda=L / \tilde{L}$, and $\eta_{1,2}=k_{1,2} \tilde{A}_{0}^{2} / \rho$, the boundary conditions are

$$
\begin{gathered}
\alpha(0)=\alpha(\lambda)=1 \\
p_{1}=p(0)=p_{\mathrm{R}}-\frac{1}{2} \mathrm{u}^{2}(0)-\eta_{1} u^{2}(0) \\
p_{2}=p(\lambda)=\eta_{2} u^{2}(\lambda)
\end{gathered}
$$

\subsection{Parameter values}

We would like to compare the results of this theory with experimental findings. Unfortunately, few experimentalists have recorded details of all the relevant parameters of their collapsible tube system, so it is not possible to give quantitative comparisons with many of the observations of the breakdown of steady flow. Like Cancelli \& Pedley (1985), therefore, we limit our comparison to three experiments: Conrad (1969), Bonis \& Ribreau (1978) and Bertram (1986) (see also Bertram 1987 and Bertram et al. 1989).

Bertram (1986, 1987) used a long, thick-walled tube, and the dimensional parameters took the following values: $D_{0}=12.7,17.5$ or $19.1 \mathrm{~mm}$; wall thickness $h=2.4 \mathrm{~mm} ; L=230 \mathrm{~mm} ; K_{p}=11.9$ or $27.15 \mathrm{kPa}$; total $\tilde{T}=3.1,22.7$ or $42.3 \mathrm{~N}$, so $T$ 
ranged from 52 to $1060 \mathrm{~N} \mathrm{~m}^{-1}$. Thus the range of values of lengthscale $\tilde{L}$ was 6.0 to $33.6 \mathrm{~mm}$ and the dimensionless length $\lambda$ varied between 6.84 and 38.2. Other nondimensional quantities are taken to be $k=45$ (measured from Bertram's tube-law graph for the $12.7 \mathrm{~mm}$ diameter tube - this value is close to the value $k=1.5\left(D_{0} / h\right)^{2}$ deduced from linear elasticity theory), and $\eta_{2}=0.014$ to $25.2, \eta_{1}=0.007$ (from data in Bertram 1986, Bertram et al. 1989). We note that $\tilde{L} / D_{0}$ was not large, varying between 0.32 and 2.65 , which is likely to limit the quantitative agreement with his experiments; other limitations arise from the use of a 'thin-membrane' model for the elastic behaviour of a thick-walled tube, and from the neglect of friction in such a long tube.

Bonis \& Ribreau (1978) used a not quite circular tube of average diameter $D_{0}=12 \mathrm{~mm}$, length $L=0.5 \mathrm{~m}$, wall thickness $h=0.31 \mathrm{~mm}$ and Young's modulus $E=1.19 \mathrm{MPa}$, giving $K_{p}\left[=E\left(2 h / D_{0}\right)^{3} / 12\left(1-\sigma^{2}\right)\right.$ where $\sigma \approx 0.5$ is the Poisson's ratio] $=18.1 \mathrm{~Pa}$ and $k \approx 2250$. The authors did not record longitudinal tension, but assuming a longitudinal strain $\tilde{\epsilon}$ of $12 \%$ (Bonis \& Ribreau 1981), and using linear theory $(T=\tilde{\epsilon} E h)$ gives $\tilde{T}=1.67 \mathrm{~N}$ and hence $\tilde{L}=0.17 \mathrm{~m}$. Thus $\tilde{L} / D_{0}$ is quite large in this case, a consequence of the thin wall of the tube which makes $K_{p}$ much smaller than in Bertram's experiment, and the dimensionless length $\lambda=2.9$. These authors do not record the upstream or downstream resistance.

Conrad (1969) also used a thin-walled tube, of rather a short length; the parameter values quoted in his paper were $D_{0}=12.7 \mathrm{~mm}, L=89 \mathrm{~mm}, h=0.93 \mathrm{~mm}$, $E=0.16 \mathrm{MPa}$, giving $K_{p}=55.8 \mathrm{~Pa}, k=2.80$. He gave no information about longitudinal tension or extension. Assuming $\tilde{\epsilon}=0.2$ gives $\tilde{T}=1.2 \mathrm{~N}, \tilde{L}=82 \mathrm{~mm}$, and hence $\lambda=1.1$. This gives $\tilde{L} / D_{0}=6.5$, which is not large enough for us to expect very good quantitative agreement between theory and experiment. Conrad records downstream resistance, giving $\eta_{2}=109$ to 8710 , but not upstream. In a personal communication in 1981, Conrad reported that the quoted value of $h$ was a misprint for $0.093 \mathrm{~mm}$, in which case $K_{p}$ would be a factor of $10^{3} \mathrm{smaller}, k$ a factor $10^{2}$ greater, $\tilde{L}$ a factor of 10 larger and hence $\lambda=0.11$. Both sets of parameter values are used in $\$ 5.3$ below.

Thus, in making comparisons with experiment we need to consider values of $\lambda$ between 0.1 and 40 . In each case we also arbitrarily consider both $\chi=0.2$ and $\chi=0.5$.

\section{Fully attached flow}

In this section we neglect all energy dissipation within the collapsible segment, to identify the conditions under which steady flows can exist. In a sense this merely repeats the analysis of Reyn (1987), but it is easier to interpret since the tube elasticity relation is much less complicated.

In non-dimensional variables, the mass and momentum conservation equations are

$$
\begin{gathered}
(u \alpha)_{x}=0 \\
u u_{x}=-p_{x}
\end{gathered}
$$

These can both be integrated with respect to $x$ :

$$
\begin{gathered}
Q=u \alpha, \\
H=\frac{1}{2} u^{2}+p
\end{gathered}
$$


where $Q$, the dimensionless flow rate, and $H$ are constants. From (2.18)

where

$$
p=p_{\mathrm{e}}+\mathscr{P}(\alpha)-\frac{1}{2} \alpha_{x x},
$$

$$
\mathscr{P}(\alpha)= \begin{cases}1-\alpha^{-\frac{3}{2}} & \text { for } \alpha \leqslant 1 \\ k(\alpha-1) & \text { for } \alpha>1\end{cases}
$$

The boundary conditions are

$$
\begin{gathered}
\alpha(0)=\alpha(\lambda)=1, \\
p_{1}=p_{\mathrm{R}}-\left(\frac{1}{2}+\eta_{1}\right) Q^{2}=p_{\mathrm{e}}-\frac{1}{2} \alpha_{x x}(0), \\
p_{2}=\eta_{2} Q^{2}=p_{\mathrm{e}}-\frac{1}{2} \alpha_{x x}(\lambda) .
\end{gathered}
$$

Using (3.3) and (3.4), it is clear from (3.7) that $p_{1}=p_{2}$; with no dissipative terms in the governing equations we expect no pressure drop along the collapsible segment. It then follows that

$$
p_{\mathrm{R}}=\left(\frac{1}{2}+\eta_{1}+\eta_{2}\right) Q^{2} .
$$

Defining another constant $P$, given by

$$
P=p_{\mathrm{e}}-p_{2},
$$

we substitute (3.3) and (3.5) into (3.4), and using the downstream boundary condition (3.9) to eliminate $H$ this gives the following second-order differential equation for $\alpha$

$$
\frac{1}{2} \alpha_{x x}=P+\frac{1}{2} Q^{2}\left(\frac{1}{\alpha^{2}}-1\right)+\mathscr{P}(\alpha) .
$$

We define

$$
f\left(Q^{2} ; \alpha\right)=\frac{1}{2} Q^{2}\left(\frac{1}{\alpha^{2}}-1\right)+\mathscr{P}(\alpha) ;
$$

$f$ has a minimum in $0 \leqslant \alpha \leqslant 1$ provided

$$
\frac{2}{3} Q^{2} \leqslant 1 .
$$

Since $c^{2}=\alpha \mathscr{P}^{\prime}(\alpha)=\frac{3}{2} \alpha^{-\frac{3}{2}}$ for $0<\alpha \leqslant 1$, this means that the flow speed entering (or leaving) the tube is subcritical: $u / c \leqslant 1$ when $\alpha=1$. The minimum value is

$$
\begin{aligned}
f_{\min }=f\left(Q^{2} ;\left(\frac{2}{3} Q^{2}\right)^{2}\right) & =-\left[\frac{1}{2} Q^{2}+\frac{1}{4}\left(\frac{2}{3} Q^{2}\right)^{-3}-1\right] \\
& =-\hat{P}^{\dagger}(Q),
\end{aligned}
$$

say. $f(\alpha)$ is monotonic decreasing in $0<\alpha<1$ for supercritical entry flow, and is monotonic increasing in $\alpha>1$ provided $Q^{2}<k$. There is a second minimum at $\alpha=\left(Q^{2} / k\right)^{\frac{1}{3}}$ if $Q^{2}>k$, where $f$ takes the value

$$
\begin{aligned}
f_{\min }=f\left(Q^{2} ;\left(\frac{Q^{2}}{k}\right)^{\frac{1}{3}}\right) & =-k\left[1+\frac{1}{2}\left(\frac{Q^{2}}{k}\right)-\frac{3}{2}\left(\frac{Q^{2}}{k}\right)^{\frac{1}{2}}\right] \\
& =-\hat{P}^{\ddagger}(Q),
\end{aligned}
$$

say. Figure 3 shows some graphs of $f ; \hat{P}^{\dagger}(Q)$ and $\hat{P}^{\ddagger}(Q)$ are shown on figure 4 .

We can examine the trajectories in the $\left(\alpha, \alpha_{x}\right)$-phase plane of the solutions of (3.12). Let $X=\alpha, Y=\alpha_{x}$. Then

$$
\left.\begin{array}{rl}
\dot{X} & =Y, \\
\frac{1}{2} \dot{Y} & =P+f\left(Q^{2} ; X\right),
\end{array}\right\}
$$

where a dot denotes differentiation with respect to $x$. 

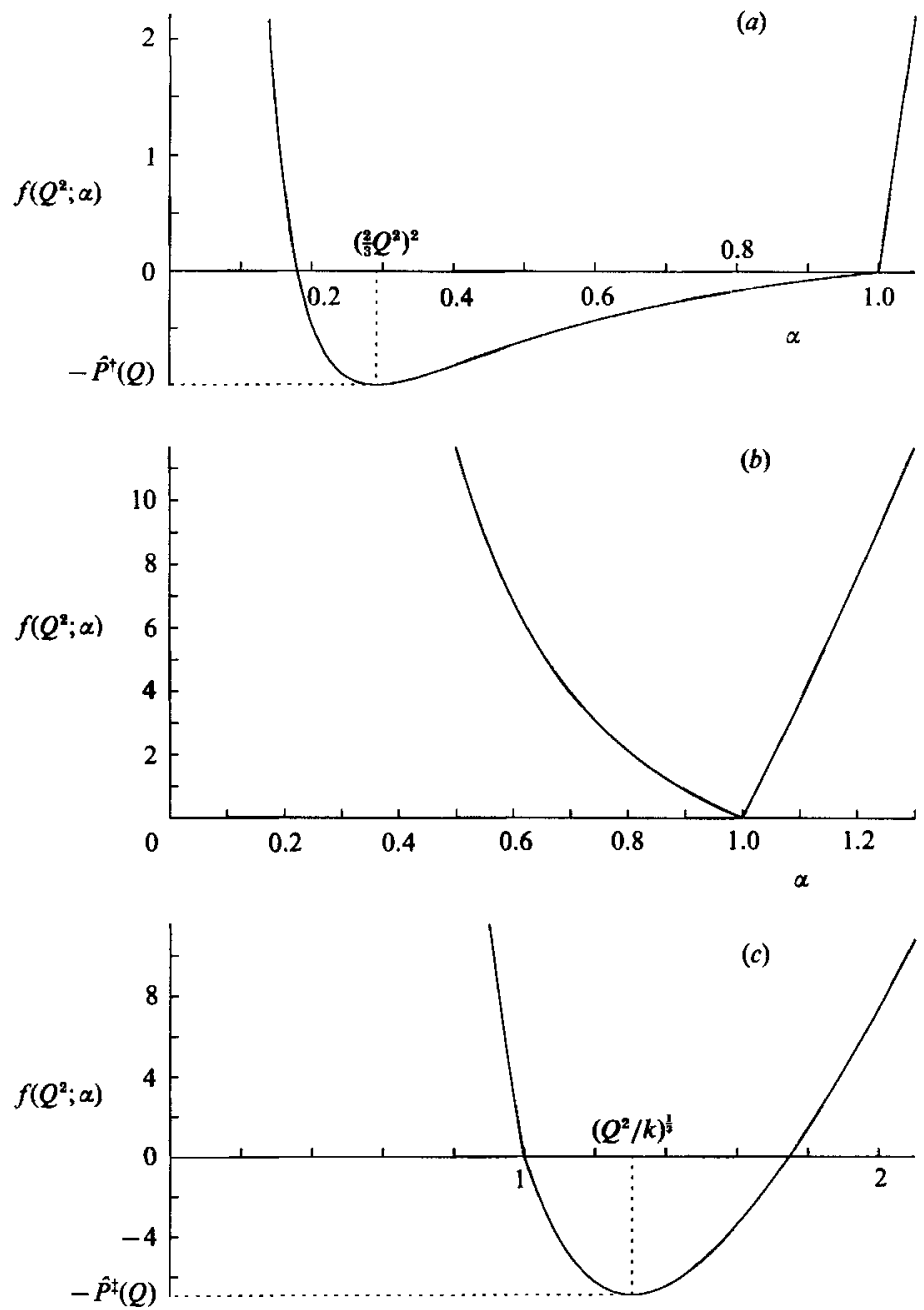

Figure 3. The function $f\left(Q^{2} ; \alpha\right)$ plotted against $\alpha$ for three values of the parameter $Q$ : (a) $0<Q<(3 / 2)^{\frac{1}{2}} ;(b)(3 / 2)^{\frac{1}{2}}<Q<k^{\frac{1}{2}} ;(c) k^{\frac{1}{2}}<Q$.

The system possesses singular points at $Y=0$ and at values of $X$ such that $f\left(Q^{2} ; X\right)=-P$. We thus see from figure 3 that there are either no singular points, when $-P$ is less than $f_{\min }$, or two when $-P>f_{\min }$ (or one, when either $-P=f_{\min }$ or $Q=0$ ). When there are two singular points, the one with the lower value of $X$ is a centre $X_{\mathrm{c}}(Q, P)$ (since $f^{\prime}(X)<0$ ) and the other is a saddle point $X_{\mathrm{s}}(Q, P)$. The functions $X_{\mathrm{s}}(Q, P)$ and $\mathrm{X}_{\mathrm{c}}(Q, P)$ are plotted as functions of $P$ for three representative values of $Q$ in figure 5. Typical phase planes are shown in figure 6; a complete, detailed discussion is given below.

Whenever the parameters $(Q, P)$ take values for which $X_{\mathrm{s}}$ and $X_{\mathrm{c}}$ exist, we may define $A_{\mathrm{s}}(Q, P)$ as the point of intersection of the saddle-point trajectory and the $X$-axis, satisfying $0<A_{\mathrm{s}}<X_{\mathrm{c}}$ (see, for example, figure $6 a \mathrm{i}$ ). $A_{\mathrm{s}}$ is given by

$$
\int_{A_{\mathrm{s}}}^{X_{\mathrm{s}}} \dot{Y} \mathrm{~d} X=0,
$$

and its dependence on $Q$ and $P$ is demonstrated in figure 5 . 


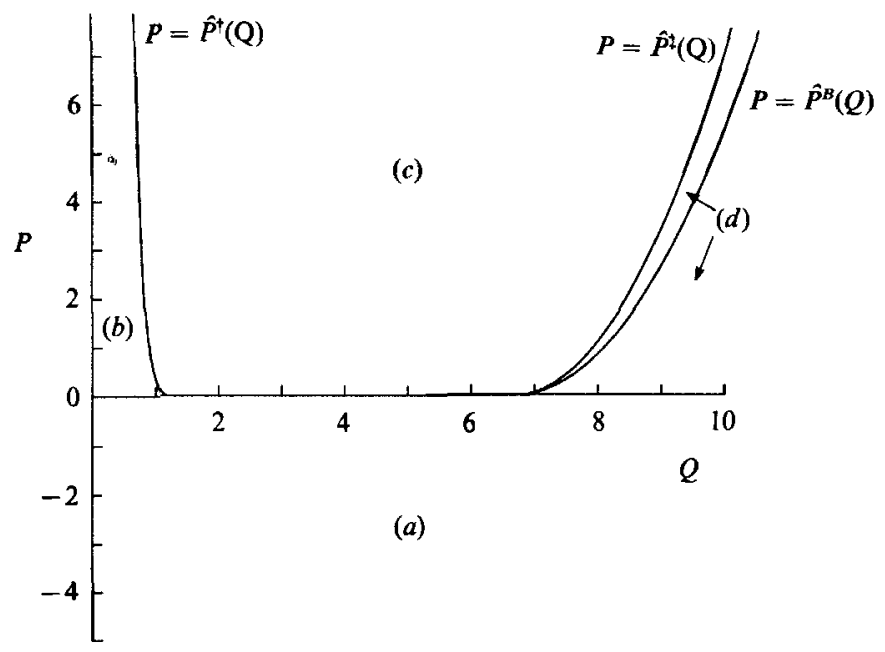

FIGURE 4. $(Q, P)$ parameter space, divided into regions $(a),(b),(c)$ and $(d)$ by the curves $P=\hat{P}^{\dagger}(Q), P=\hat{P}^{\ddagger}(Q), P=\hat{P}^{B}(Q)$ and $P=0 .(k=45$ was used in calculating these curves.)

Consider the trajectory in figure $6(c)$ (i) which passes from 1 to 2 to 3 . It corresponds to a solution (shown in figure $7 a$ ) for a tube which is collapsed at its mid-point, where the cross-sectional area takes an extremum value $A$. Likewise a distended tube, such as that in figure $7(b)$, is represented by the trajectory numbered $1,2,3$ in figure $6(e)(\mathrm{i})$. $(3,4,1$, represents a collapsed tube.) It is convenient to associate particular solutions for given $Q, P$ with the corresponding values of $A$.

Multiplying (3.12) by $\alpha_{x}$ and integrating with respect to $x$ gives:

$$
\frac{1}{4} \alpha_{x}^{2}=g(\alpha)+\text { constant }
$$

where

$$
g(\alpha)=P(\alpha-1)+\frac{1}{2} Q^{2}\left(2-\frac{1}{\alpha}-\alpha\right)+\int_{1}^{\alpha} \mathscr{P}(\alpha) \mathrm{d} \alpha .
$$

At $\alpha=A, \alpha_{x}=0$, so

$$
\frac{1}{4} \alpha_{x}^{2}=g(\alpha)-g(A)
$$

A necessary condition for the existence of a solution is $g(\alpha)-g(A) \geqslant 0$ for all $\alpha$ between 1 and the extremum value $A$. If $A<1$, we may define

$$
I(A ; Q, P)=\int_{A}^{1} \frac{\mathrm{d} \alpha}{[g(\alpha)-g(A)]^{\frac{1}{2}}} ;
$$

if $A>1, I$ is simply defined with the limits of integration reversed. Thus for a collapsed tube, $\frac{1}{2} I$ corresponds to integrating the inverse square root of (3.21) along a trajectory from point 2 in figure $6(c)(i)(X=A)$ to point $3(X=1)$ which, by symmetry, corresponds to integrating along half the length of the tube. We therefore have

$$
\lambda=I(A ; Q, P) \text {. }
$$

The independent governing parameters of the problem are $Q$ and $P$. $(Q, P)$-space is divided naturally into four regions, as shown on figure 4; figures $6(a)-6(e)$ show the corresponding types of behaviour. 

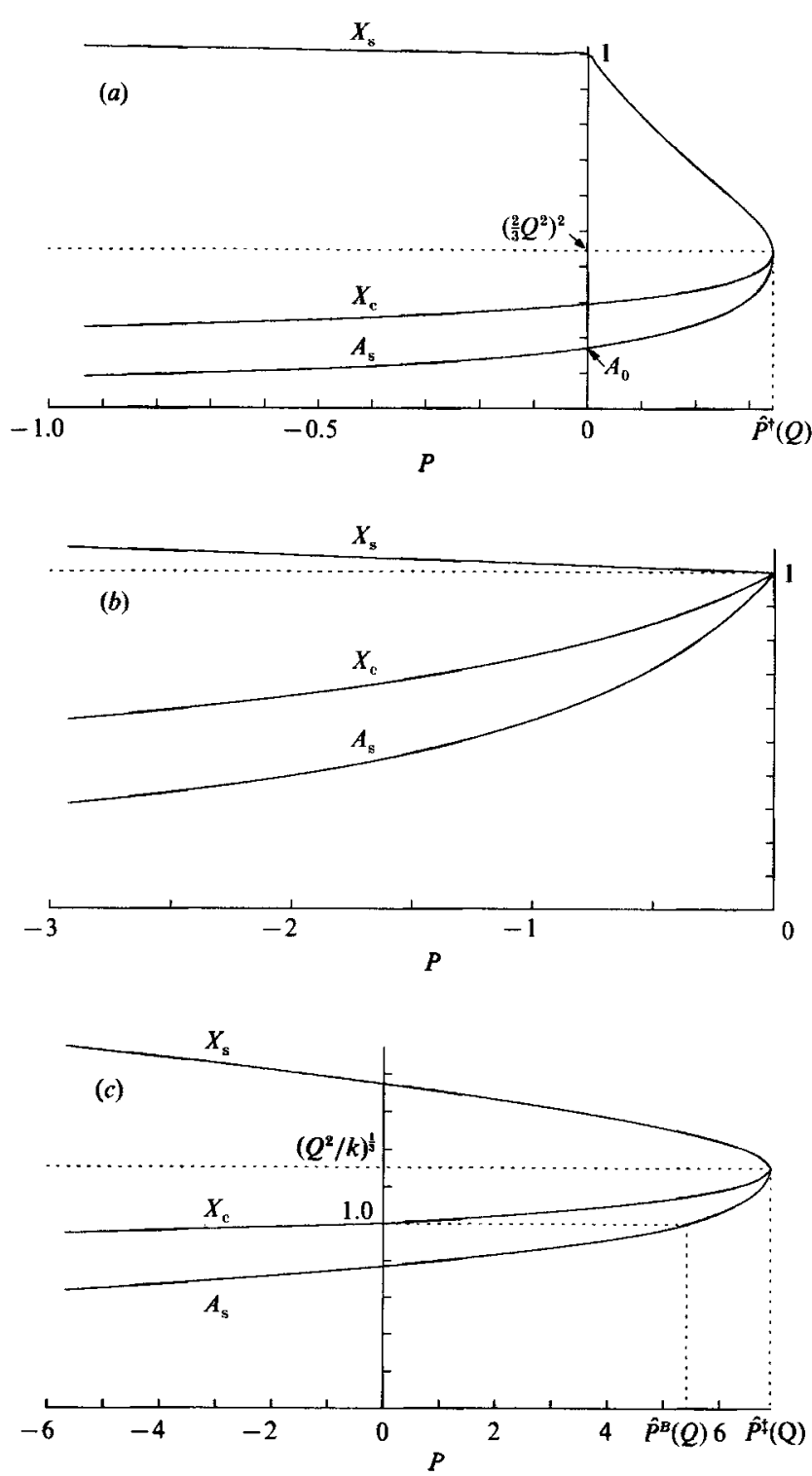

Figure 5. The functions $X_{\mathrm{s}}(Q, P), X_{\mathrm{c}}(Q, P)$ and $A_{\mathrm{s}}(Q, P)$ plotted against $P$ for three values of $Q$ : (a) $0<Q<(3 / 2)^{\frac{1}{2}} ;(b)(3 / 2)^{\frac{1}{2}}<Q<k^{\frac{1}{2}} ;(c) k^{\frac{1}{2}}<Q$.

(a) $0<Q, P<0$

Whenever $P<0$, we see from figure 5 that the phase plane contains two singular points, a centre $X_{\mathrm{c}}(Q, P)$ with $X_{\mathrm{c}}<1$ and a saddle point $X_{\mathrm{s}}(Q, P)$ with $X_{\mathrm{s}}>1$; such a phase plane is shown in figure $6(a)$ (i). Trajectories inside the closed, dotted loop (which passes through $\left(A_{1}, 0\right)$ and $(1,0)$ ) do not correspond to possible solutions since $X \neq 1$ on them. Thus solutions for which the tube is collapsed are possible only for $A<A_{1}$; solutions in which the tube is distended are possible for $1<A<X_{\mathrm{s}}$. Following Reyn (1987), in figure $6(a)$ (ii) we also plot the values of 1 , that is to say, dimensionless tube length $\lambda$ corresponding to the different permitted values of $A$. (We also use his notation to label trajectories which cross $X=1$ when $Y>0(\mathrm{U})$ and 

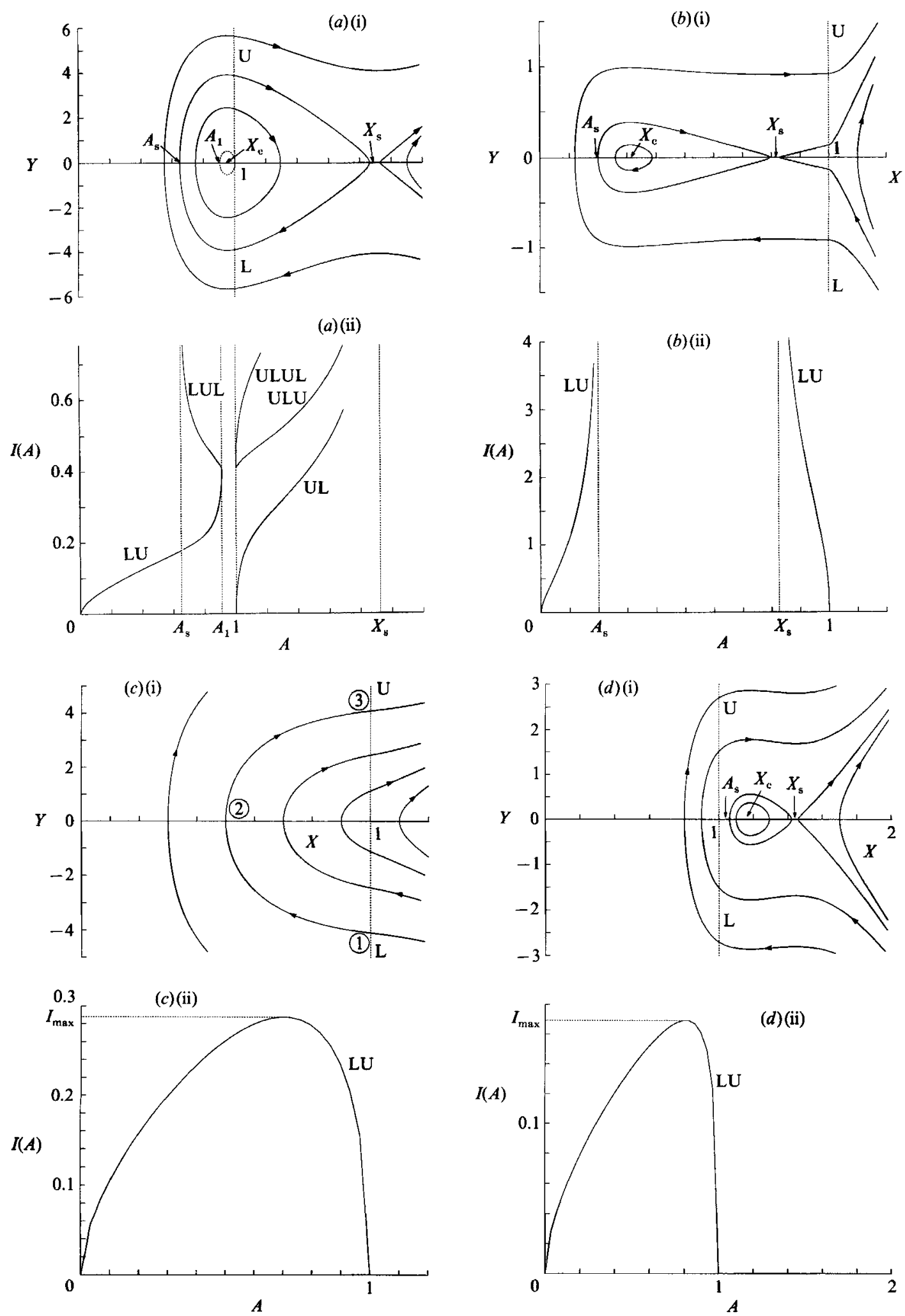

Frgure $6(a)(\mathrm{i}, \mathrm{ii}),(b)(\mathrm{i}, \mathrm{ii}),(c)(\mathrm{i}, \mathrm{ii}),(d)(\mathrm{i}, \mathrm{ii})$. For caption see facing page. 

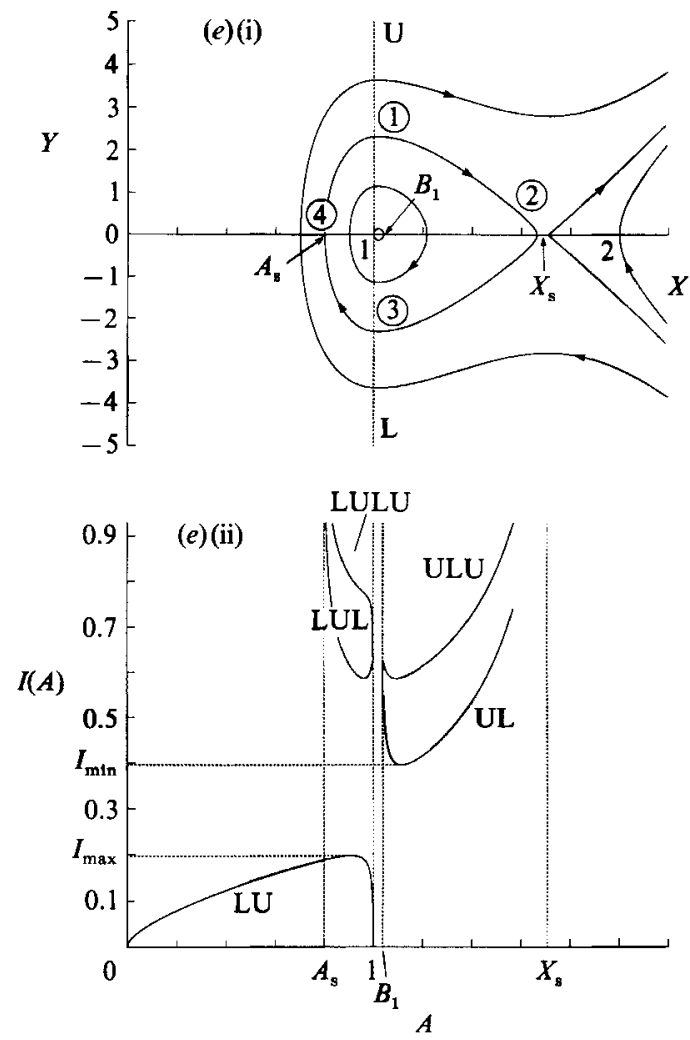

Figure 6. (i) Trajectories in the $\left(\alpha, \alpha_{x}\right)$ phase plane $\left(X \equiv \alpha\right.$ and $\left.Y \equiv \alpha_{x}\right)$, each a possible tube configuration with the flow fully attached. To represent a tube held open at both ends, they must cross $\alpha=1$ (dotted) twice, with $\alpha_{x}<0$ (L) or $\alpha_{x}>0$ (U). (ii) The corresponding graphs of $I(A)$, which relate the change in the parameter $x$ along a trajectory between intersections with $\alpha=1$, to the extremum area of the tube shape represented by that trajectory. Parameter values are as follows: $(a) Q=10, P=-5$ (region $a$ of figure 4 ); $(b) Q=1, P=0.1$ (region $b) ;(c) Q=5, P=5$ $($ region $c) ;(d) Q=10, P=6$ (region $d) ;(e) Q=10, P=1$ (also region $d) ; k=45$ in all cases.

$X=1$ when $Y<0(\mathrm{~L})$, so that, for example, trajectory $1,2,3$ in figure $6(c)(\mathrm{i})$ is denoted $\mathrm{LU}$, whereas trajectory $1,2,3$ in figure $6(e)(\mathrm{i})$ is represented by $\mathrm{UL}$.)

As one might expect, whenever there is negative transmural pressure, solutions exist for all values of $\lambda$ with the tube distended (see the branch of figure $6(a)$ (ii) corresponding to UL trajectories). Interestingly, the solutions for $A>1$ are not unique if $\lambda$ is sufficiently large (e.g. ULU and ULUL in figure 6aii): the model predicts that the tube may take up a configuration in which $\alpha$ has alternating extrema greater or less than 1 (the definition of $I$ is here extended to allow repeated passage of a trajectory across the $X$-axis); such non-uniqueness was also found by Reyn (1987). (To ensure that the pressure and its gradient are continuous at points along the tube where $\alpha=1$, at which the tube law has discontinuous gradient, $\alpha_{x x}$ must be continuous but $\alpha_{x x x}$ is discontinuous.)

There are similar non-unique solutions with $A<A_{1}$, as long as $\lambda$ is sufficiently large. More surprisingly, there is a collapsed solution with $\alpha \leqslant 1$ everywhere (LU), as well as the expected distended one with $\alpha \geqslant 1$ everywhere, for sufficiently small $\lambda$; the flow corresponding to this collapsed state is highly supercritical along most of the collapsed segment. This apparently anomalous solution no longer exists when allowance is made for energy loss (see $\$ 4$ below). 

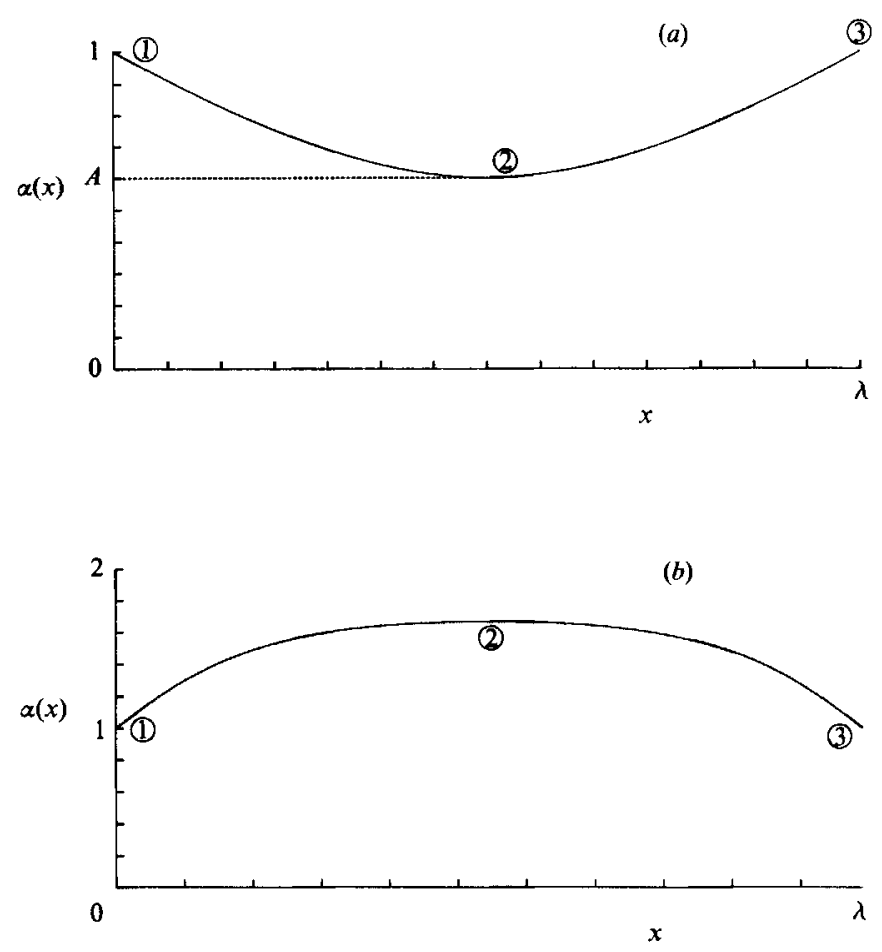

Fiaure 7. Two typical tube shapes when the flow is fully attached: $(a)$ the collapsed tube corresponds to the trajectory in $\left(\alpha, \alpha_{x}\right)$ phase space numbered $1,2,3$ in figure $6(c)(i) ;(b)$ the trajectory numbered $1,2,3$ in figure $6(e)$ (i) represents this swollen configuration.

(b) $0<Q<(3 / 2)^{\frac{1}{2}}, 0 \leqslant P \leqslant \hat{P}^{\dagger}(Q)$

For subcritical entry flow and low pressures figure $5(a)$ shows us that we have $0<X_{\mathrm{c}} \leqslant\left(\frac{2}{3} Q^{2}\right)^{2} \leqslant X_{\mathrm{s}} \leqslant 1$; the phase plane is as shown in figure $6(b)(\mathrm{i})$. Two solutions for a collapsed tube exist for all values of $\lambda$, with different values of $A: 0<A<A_{\mathrm{s}}$ and $X_{\mathrm{s}}<A<1$. The two branches of $I$ (see figure $6 \mathrm{bii}$ ) are separated by a region which contains the point $A=\left(\frac{2}{3} Q^{2}\right)^{2}$. Now the speed index (the ratio of flow speed to the speed of propagation of pressure waves in the tube wall) is given by

$$
\frac{u}{c}=\left(\frac{2}{3} Q^{2} / \alpha^{\frac{1}{2}}\right)^{\frac{1}{2}} \quad \text { for } \alpha \leqslant 1 .
$$

Thus solutions on the right-hand branch represent flow that is everywhere subcritical, while those on the left-hand branch correspond to flow that is subcritical on entry but supercritical at the constriction. When $P=0$ this right-hand branch coincides with the vertical line $X_{\mathrm{s}}=1$. A cylindrical-tube solution emerges in this case for all tube lengths.

(c) $0<Q<(3 / 2)^{\frac{1}{2}}, \hat{P}^{\dagger}(Q)<P$ or $(3 / 2)^{\frac{1}{2}} \leqslant Q<k^{\frac{1}{2}}, 0<P$ or $k^{\frac{1}{2}}<Q, \hat{P}^{\dagger}(Q)<P$ In these cases the phase plane has no singular points (figure $6 \mathrm{ci}$ ); $I(A)$ is well defined for all $A \in[0,1]$, vanishes as $A \rightarrow 0$ and has a finite upper bound $I_{\max }(Q, P)$, as shown in figure $6(c)$ (ii). Thus for $\lambda>I_{\max }$ no solutions exist, while for $0<\lambda<I_{\max }$ two solutions for a collapsed tube are possible. 


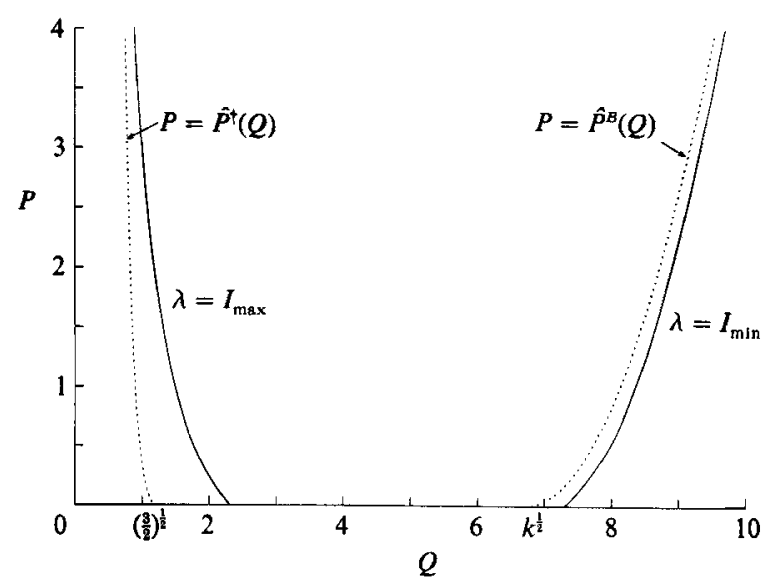

Figure 8. The contours $\lambda=I_{\max }(Q, P)$ and $\lambda=I_{\min }(Q, P)$ plotted for $\lambda=1, k=45$. Each lies to the right of $P=\hat{P}+(Q)$ and $P=\hat{P}^{B}(Q)$ respectively. For this tube length, there are no steady solutions with the flow fully attached for $(Q, P)$ between these contours and $P=0$.

\section{(d) $k^{\frac{1}{2}} \leqslant Q, 0 \leqslant P \leqslant \hat{P}^{\ddagger}(Q)$}

In this case both a saddle point $X_{\mathrm{s}}$ and a centre $X_{\mathrm{c}}$ exist in $X \geqslant 1$, and thus $A_{\mathrm{s}}$ also exists as shown in figure $5(c)$. Note that $A_{\mathrm{s}}=1$ when $P$ takes a value $\hat{P}^{B}(Q)$, say, where $0<\hat{P}^{B}(Q)<\hat{P}^{\ddagger}(Q)$. When this is the case, we have (from (3.18)) $g\left(X_{\mathrm{s}}\right)-g(1)=0$, and from our definition of $g(3.20)$ we have $g(1)=0$; further, since $f\left(X_{\mathrm{s}}\right)=0, g^{\prime}\left(X_{\mathrm{s}}\right)=0$. Thus $g\left(X_{\mathrm{s}}\right)=0$ has a double root which is not equal to 1 , and from this we deduce that $X_{\mathrm{s}}=Q / k^{\frac{1}{2}}$ and

$$
\hat{P}^{B}(Q)=\frac{1}{2}\left(Q-k^{\frac{1}{2}}\right)^{2} .
$$

$\hat{P}^{B}(Q)$ divides this region of parameter space as shown on figure 4 . If $\hat{P}^{B}(Q)<P \leqslant \hat{P}^{\ddagger}(Q)$, the saddle-point trajectory lies entirely in $X>1$ and $I(A)$ is of the same form as in case $(c)$ (see figures $6 d$ i and ii). For $0 \leqslant P<\hat{P}^{B}(Q)$, we have $A_{\mathrm{s}}<1$ and phase planes such as that shown in figure $6(e)$ (i). We may define $B_{1}$ analogously to $A_{1}$, i.e. the trajectory through $(1,0)$ passes through $\left(B_{1}, 0\right)$, where $B_{1}>1$. I $(A)$ is as shown in figure $6(e)$ (ii). In addition to two LU solutions which exist for sufficiently short tubes $\left(\lambda<I_{\max }\right)$, further solutions arise provided $\lambda$ is sufficiently large, again characterized by alternate dilation and contraction down the length of the tube. Note in particular that the curve representing a completely dilated tube (UL) has a minimum value, $I_{\min }$, say, for some value of $A$ in $\left(B_{1}, X_{\mathrm{s}}\right)$, so that for

no solutions exist.

$$
I_{\max }<\lambda<I_{\min }
$$

Therefore for a tube of given dimensionless length $\lambda$, the contour

$$
\lambda=I_{\max }(Q, P)
$$

separates the region of $(Q, P)$-space for which solutions exist with the tube collapsed $(\alpha \leqslant 1$ everywhere) from that in which they do not. Such a contour is shown in figure 8. Beneath the curve $P=\hat{P}^{\dagger}(Q)$ (shown dotted in the figure), there exist two collapsed solutions for all lengths of tube. The contour is to be found above and to the right of this curve. In addition, for $(Q, P)$ lying to the right of the contour $\lambda=I_{\min }$, (also shown on figure 8), or for $P<0$, a further class of solutions exists such that the tube may be completely dilated, or have alternating maxima and minima. 


\section{Separated flow}

In this discussion we shall determine how energy loss through flow separation affects the parameter values for which steady solutions exist, and we shall discuss the behaviour of these solutions. Since we are interested only in tubes that are constricted at some point, we shall henceforth assume that $P>0$ (except at the end of $\$ 4.3$ ).

\subsection{The governing equations}

Following the discussion of $\$ 2.1$, the momentum equation for flow that separates downstream of a constriction is

$$
\chi u u_{x}=-p_{x}
$$

where $\chi=1$ upstream of the separation point $\left(x=X_{0}\right)$ and $\chi$ is a constant, $0<\chi<1$, downstream of it. In general the discontinuity in $\chi$ causes a jump in the pressure gradient, as was the case in the calculations of Cancelli \& Pedley (1985) who used the size of the adverse pressure gradient as the criterion determining the position of the separation point. However, since we are using a simplified criterion - that the separation point is the point at which $\alpha$ takes its minimum area $A$, so that $\alpha_{x}\left(X_{0}\right)=u_{x}\left(X_{0}\right)=0$ - we in fact have a continuous pressure gradient at $x=X_{0}$. As a result, $\alpha, \alpha_{x}, \alpha_{x x}$ and $\alpha_{x x x}$ are continuous across the separation point.

Integration of the conservation equations (3.1) and (4.1) gives

$$
\begin{aligned}
& Q=u \alpha \quad \text { for } 0 \leqslant x \leqslant \lambda, \\
& \frac{1}{2} u^{2}+p=H_{1} \quad \text { (constant) for } 0 \leqslant x \leqslant X_{0} \text {, } \\
& \frac{1}{2} \chi u^{2}+p=H_{2} \quad \text { (constant) for } X_{0} \leqslant x \leqslant \lambda \text {. }
\end{aligned}
$$

Using the boundary conditions

$$
\begin{gathered}
\alpha(0)=1, \quad \alpha(\lambda)=1, \\
p_{1}=p_{\mathrm{e}}-\hat{P}, \text { say }, \quad p_{2}=p_{\mathrm{e}}-P,
\end{gathered}
$$

(4.2), (4.3) and (4.4) give

$$
\frac{1}{2} \alpha_{x x}= \begin{cases}\hat{P}+\frac{1}{2} Q^{2}\left(\frac{1}{\alpha^{2}}-1\right)+\mathscr{P}(\alpha) \quad \text { when } 0 \leqslant x \leqslant X_{0} \\ P+\frac{1}{2} \chi Q^{2}\left(\frac{1}{\alpha^{2}}-1\right)+\mathscr{P}(\alpha) \text { when } X_{0} \leqslant x \leqslant \lambda .\end{cases}
$$

Since $\alpha_{x x}$ is continuous at $x=X_{0}$, where $\alpha=A$,

$$
\hat{P}(A)=P-\frac{1}{2}(1-\chi) Q^{2}\left(\frac{1}{A^{2}}-1\right),
$$

i.e. the pressure drop along the collapsible segment is

$$
p_{1}-p_{2}=\frac{1}{2}(1-\chi) Q^{2}\left(\frac{1}{A^{2}}-1\right) .
$$

As before solutions of (4.7) are best described by examining their trajectories in the $(X, Y)$-phase plane, where $X=\alpha$ and $Y=\alpha_{x}$. The separation point of a collapsed tube corresponds to the point $X=A, Y=0$ (see figure $9 a$ ). The form of the tube 


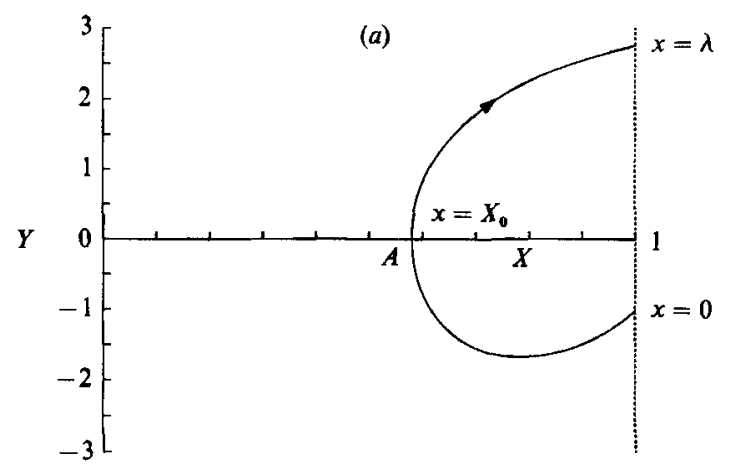

(b)

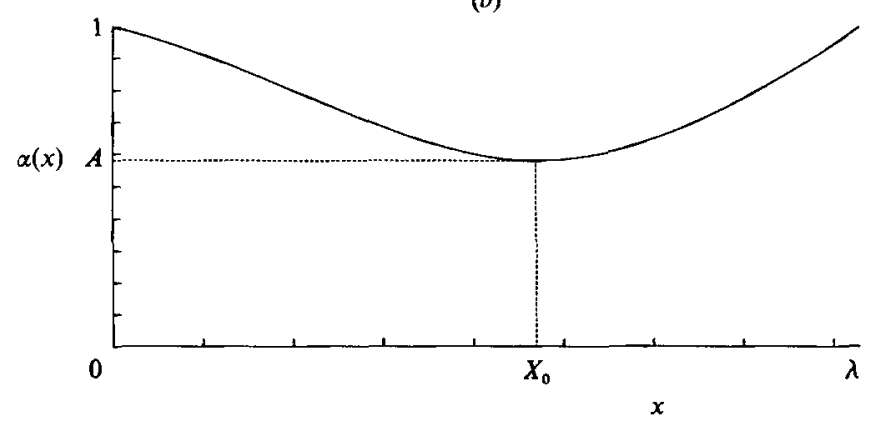

Figure 9. (a) A trajectory in $\left(\alpha, \alpha_{x}\right)$ phase space $\left(X \equiv \alpha\right.$ and $\left.Y \equiv \alpha_{x}\right)$ representing a collapsed tube with separation occuring at $(A, 0)$, the point of minimum area. The corresponding tube shape is shown in $(b)$. Parameter values are $Q=P=5, k=45$ and $\chi=0.5$.

downstream of this point is represented by the trajectory calculated by integration over $x \in\left[X_{0}, \lambda\right]$ of

$$
\left.\begin{array}{c}
\dot{X}=Y, \\
\frac{1}{2} \dot{Y}=P+\frac{1}{2} \chi Q^{2}\left(\frac{1}{X^{2}}-1\right)+\mathscr{P}(X) .
\end{array}\right\}
$$

(Note that we may here use the results of $\$ 3$ but with $Q^{2}$ replaced by $\chi Q^{2}$.) To satisfy the downstream boundary condition $(4.5 b)$, the trajectory must cross $X=1$ when $x=\lambda$. Likewise, the upstream trajectory describing the tube for $x \in\left[0, X_{0}\right]$ is obtained by integrating

$$
\begin{gathered}
\dot{X}=Y, \\
\frac{1}{2} \dot{Y}=\hat{P}+\frac{1}{2} Q^{2}\left(\frac{1}{X^{2}}-1\right)+\mathscr{P}(X)
\end{gathered}
$$

backwards from the separation point. Equation (4.5a) requires this trajectory to cross $X=1$ when $x=0$. Figure $9(b)$ shows the actual shape of the tube corresponding to the trajectory in figure $9(a)$.

From (4.8), we may re-express $(4.11 b)$ as follows:

$$
\frac{1}{2} \dot{Y} \equiv P+\frac{1}{2} \chi Q^{2}\left(\frac{1}{X^{2}}-1\right)+\mathscr{P}(X)-(1-\chi) \frac{1}{2} Q^{2}\left(\frac{1}{A^{2}}-\frac{1}{X^{2}}\right) .
$$




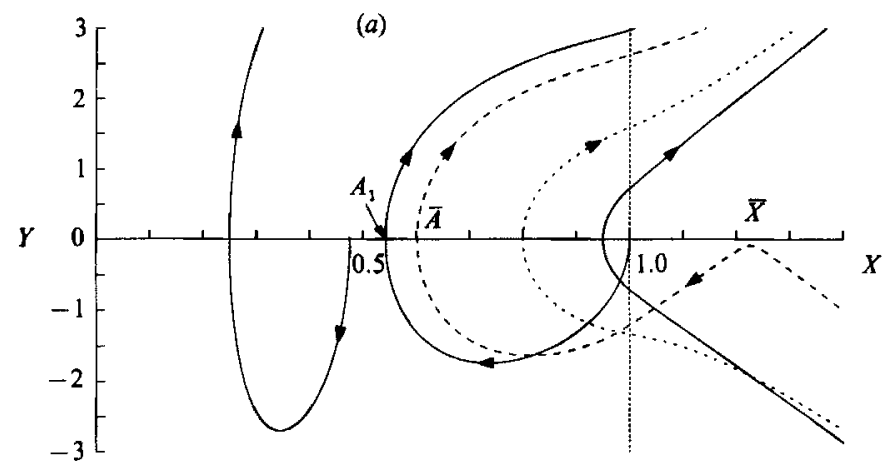

(b)
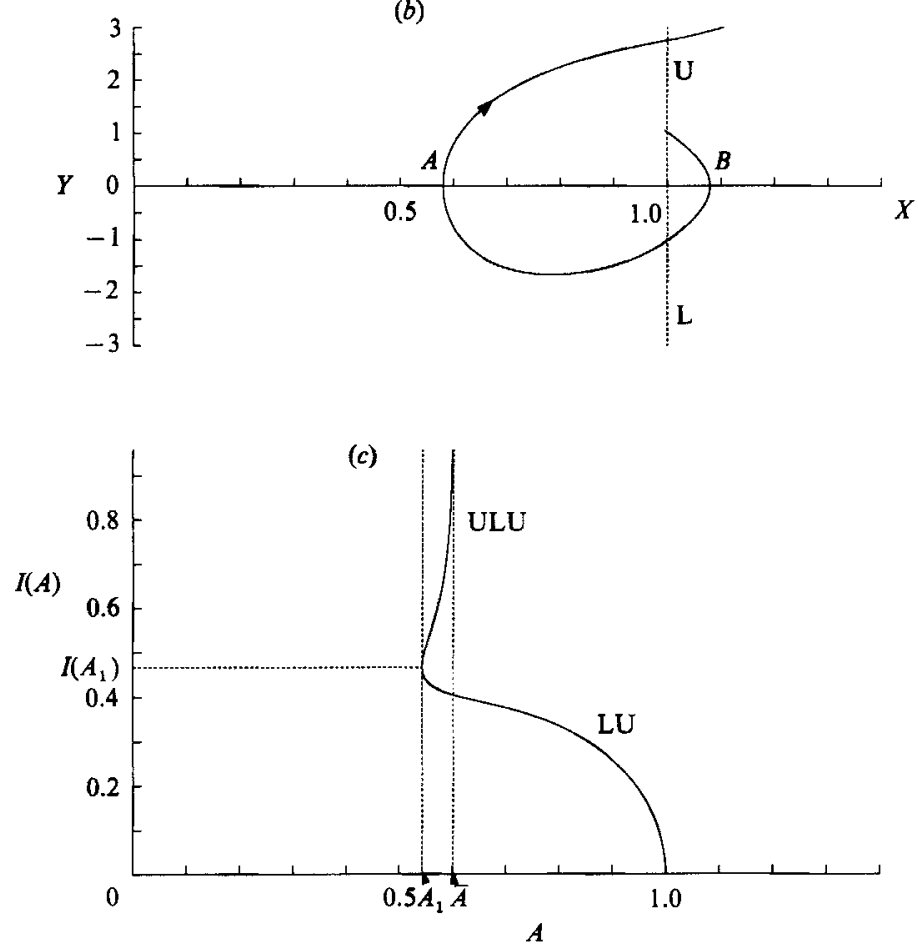

Figure 10. $(a)$ Trajectories in the $\left(\alpha, \alpha_{x}\right)$ phase plane representing tube shapes when the flow separates at the point of minimum area (along $\alpha_{x}=0$ ), for $\chi=0.5, k=45$ and $Q=P=5$ (in region $e$ of figure 11); downstream they are all governed by the same equation - this is not the case upstream. (b) A single trajectory which meets the $X$-axis with $A_{1}<A<\bar{A}$, representing a tube swollen at its upstream end and collapsed further downstream, denoted ULU. (c) The graph of $I(A)$ for these parameter values, relating tube length to minimum area.

We see that the upstream trajectory is governed by equations identical to (4.10) except for an extra term

$$
-(1-\chi) \frac{1}{2} Q^{2}\left(\frac{1}{A^{2}}-\frac{1}{X^{2}}\right)
$$

Because this term depends on $A$, different upstream trajectories will be governed by different equations, so it is likely that they may cross one another (even away from any singular points.) 
We illustrate the effect of this term with an example. Take $Q=5.0, P=\mathbf{5 . 0}$ and set $\chi=0.5$ arbitrarily. Since $\chi Q^{2}>\frac{3}{2}$, the downstream trajectories have no singular points, as shown in the upper half-plane of figure $10(a)$.

For $X>A$, the term (4.13) is negative and its effect is to bring the upstream trajectory which passes through $(A, 0)$ closer to the $X$-axis than its downstream counterpart. The influence of the term increases as $X$ moves away from $A$ and also as $A$ decreases. Thus for $A$ near 1 , upstream and downstream trajectories are not qualitatively different; as $A$ decreases (e.g. see the dotted trajectory on figure $10 a$ ) the corresponding upstream trajectories bend closer to the $X$-axis; the upstream trajectory passing through $\bar{A}$ (dashed on figure $10 a$ ) meets the $X$-axis at a saddle point at $(\bar{X}, 0)$, which for these values of $Q$ and $P$ happens to lie beyond $X=1$.

As $A$ decreases from $\bar{A}$, the effect of (4.13) is progressively greater. Upstream trajectories form loops which become tighter as $A$ becomes smaller. Figure $10(a)$ shows the trajectory through $A=A_{1}$, which passes through $(1,0)$. If $A<A_{1}$, then all upstream trajectories form loops which start and finish in $0<X<1$, making it impossible to satisfy the upstream boundary condition (4.5 $a$ ). For $A_{1}<A<\bar{A}$, we can find trajectories such as the one shown in figure $10(b)$. Starting from $X=1$, $Y<0$, passing through $X=A, Y=0$ and returning to $X=1, Y>0$, it represents a collapsed tube held open at both ends (LU in Reyn's notation). In addition, from a point on $X=1, Y>0$ we may follow a path ULU corresponding to a tube that is swollen just beyond $x=0$ but that collapses before $x=\lambda$; there is assumed to be no flow separation at $x=0$ so there is no energy loss in the distended region.

Letting $I(A ; Q, P)$ be the change in the parameter $x$ down a trajectory representing a collapsed tube, we must again satisfy

$$
\lambda=I(A ; Q, P) \text {. }
$$

For the parameter values of this example, $I(A ; Q, P)$ is not defined for all $A$ in $[0,1]$; it has the form shown in figure $10(c)$. For $A<A_{1}$, there are no trajectories that meet $X=1$ twice, as required. Its branch representing a tube that is everywhere collapsed is bounded above by $I\left(A_{1}\right)$, so that for these parameter values there is no LU-solution for a tube of length greater than $I\left(A_{1}\right)$. However, the branch representing tubes that are partially swollen and partially collapsed (ULU) tends to infinity as $A \rightarrow \bar{A}-$.

Explicitly, for a tube that is collapsed at some point along its length and held open at both ends $I$ is of the following form:

$$
I(A ; Q, P)=I_{1}+I_{2}
$$

where

$$
I_{i}=\frac{1}{2} \int_{A}^{1} \frac{\mathrm{d} \alpha}{\left[g_{i}(\alpha)-g_{i}(A)\right]^{\frac{1}{2}}} \quad \text { for } i=1,2
$$

and

$$
\left.\begin{array}{c}
g_{1}(\alpha)=\hat{P}(\alpha-1)+\frac{1}{2} Q^{2}\left(2-\frac{1}{\alpha}-\alpha\right)+\int_{1}^{\alpha} \mathscr{P}(\alpha) \mathrm{d} \alpha . \\
g_{2}(\alpha)=P(\alpha-1)+\frac{1}{2} \chi Q^{2}\left(2-\frac{1}{\alpha}-\alpha\right)+\int_{1}^{\alpha} \mathscr{P}(\alpha) \mathrm{d} \alpha .
\end{array}\right\}
$$

Again, the existence of $I$ is conditional on

$$
g_{1}(\alpha)-g_{1}(A) \geqslant 0, \quad g_{2}(\alpha)-g_{2}(A) \geqslant 0
$$

for all $\alpha$ between 1 and the minimum $A$. Note that

$$
g_{2}(\alpha)-g_{2}(A) \geqslant g_{1}(\alpha)-g_{1}(A) \text { for all } \alpha \text { in }[A, 1]
$$


so that $I_{1} \geqslant I_{2}$ and therefore the separation point $X_{0}$ lies in the downstream half of the tube. As in $\S 3$, we may extend the definition of $I$ to include situations in which the tube is dilated as well as collapsed at some point along its length.

\subsection{Properties of the phase plane}

We now determine some characteristics of the phase plane in various regions of $(Q, P)$-parameter space. The essential results will be summarized in the next section, §4.3.

The downstream trajectories are easily dealt with. From the analogous results in $\S 3((3.15)$ and $(3.16))$, we may define (for $\left.\chi Q^{2}<\frac{3}{2}\right)$

$$
P^{\dagger}(Q)=-f\left(\chi Q^{2} ;\left(\frac{2}{3} \chi Q^{2}\right)^{2}\right)
$$

and for $\chi Q^{2}>k$

$$
P^{\ddagger}(Q)=-f\left(\chi Q^{2} ;\left(\frac{\chi Q^{2}}{k}\right)^{\frac{1}{3}}\right),
$$

where $f$ is given by (3.13). $P=P^{\dagger}(Q)$ is the curve (shown on figure 11i) beneath which downstream trajectories have a saddle point $S_{\mathrm{D}}$, say, and a centre $C_{\mathrm{D}}$ in $0<X<1$ (cf. figure $6 b \mathrm{i}$ ). Above this curve, and to the left of $P=P^{\ddagger}(Q)$ (shown in figure 11ii), there are no singular points in the upper half of the phase plane. For $\chi Q^{2}>k$ and $P<P^{\ddagger}(Q), S_{\mathrm{D}}$ and $C_{\mathrm{D}}$ both exist and are found in $X>1$ (cf. figure $6 d \mathrm{i}$ ).

As mentioned at the start of this section, along a trajectory crossing a separation point $(A, 0), \alpha_{x}=\dot{X}$ and $\alpha_{x x}=\dot{Y}$ are continuous. Thus if an upstream and a downstream trajectory meet at a downstream singular point, this is also an upstream singular point. There are therefore only three types of upstream singularity corresponding to a downstream saddle point $S_{\mathrm{D}}$ or centre $C_{\mathrm{D}}$ (or to the degenerate singularity $(C S)_{\mathrm{D}}$ formed when $S_{\mathrm{D}}$ and $C_{\mathrm{D}}$ coalesce): a centre $C_{\mathrm{U}}$, a saddle $S_{\mathrm{U}}$ or a degenerate singularity $(C S)_{\mathrm{U}}$. Corresponding to $C_{\mathrm{D}}$ or $(C S)_{\mathrm{D}}$ we must always have $C_{\mathrm{U}}$, since (4.13) prevents any upstream trajectory from moving further away from the $X$-axis than its downstream counterpart.

To determine which singularities correspond to $S_{\mathrm{D}}$ for given $(Q, P)$, and also to ascertain whether upstream saddle-point trajectories such as that through $(\bar{A}, 0)$ in the example above exist or not, it is helpful to take the following approach.

Upstream trajectories are governed by $(4.11)$ with $\hat{P}(A)$ given by $(4.8)$. For given $(Q, P)$, let $A$ take some fixed value. This defines a particular $\hat{P}$, and (4.11) in turn defines an entire half-plane of upstream trajectories. (Only one of these trajectories that through $(A, 0)$ - corresponds to a possible tube configuration for these values of $Q$ and $P$.)

If $\hat{P}(A)$ is sufficiently large for there to be no singular points on this phase plane, then we can be sure the trajectory through $(A, 0)$ will be non-singular. However, if we have (say) $Q<(3 / 2)^{\frac{1}{2}}$ and $\hat{P}<\hat{P}^{\dagger}(Q)$ (defined as in (3.15)), then a centre at $\left(X_{\mathrm{c}}(Q, \hat{P}), 0\right)$ and a saddle point at $\left(X_{\mathrm{s}}(Q, \hat{P}), 0\right)$ are to be found in this phase plane (see for example figure $6 b \mathrm{i})$; likewise the saddle-point trajectory through $\left(A_{\mathrm{s}}(Q, \hat{P}), 0\right)$ will exist. The values of $A_{\mathrm{s}}, X_{\mathrm{c}}$ and $X_{\mathrm{s}}$ may be determined from figure $5(a)$, replacing $P$ with $\hat{P}$. We may then determine the nature of the trajectory $T$ through $(A, 0)$ in the $(X, Y)$-phase plane by examining the relationship between $A$ and $A_{\mathrm{s}}(Q, \hat{P}(A))$, $X_{\mathrm{c}}(Q, \hat{P}(A))$ and $X_{\mathrm{s}}(Q, \hat{P}(A))$. Figure 12 shows some possible forms of $T$. (Note that when $X_{\mathrm{c}}<A<X_{\mathrm{s}}$, the case shown in figure $12(c)$, there exist no upstream 

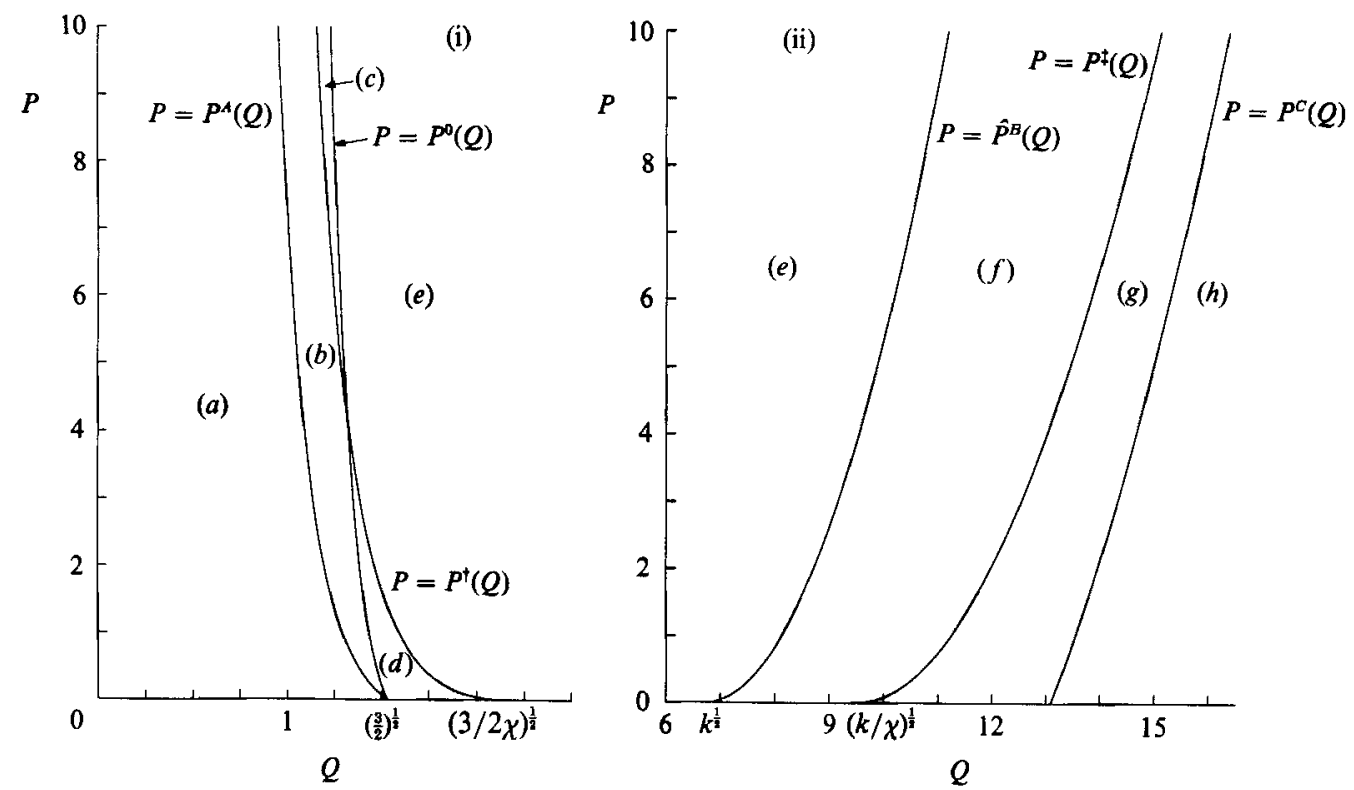

Figure 11. $(Q, P)$ parameter space divided into sections in which the corresponding $\left(\alpha, \alpha_{x}\right)$ phase plane has different characteristics: (i) divided into regions $(a-e)$ by the curves $P=P^{A}(Q)$, $P=P^{0}(Q), P=P^{\dagger}(Q)$; (ii) divided into regions $(e-h)$ by $P=\hat{P}^{B}(Q), P=P^{\ddagger}(Q)$ and $P=P^{c}(Q)$. $\chi=0.5, k=45$.

(a)

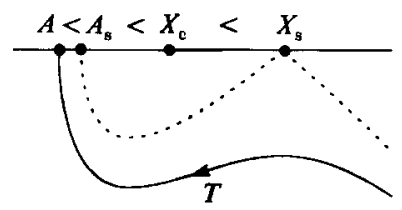

(c)

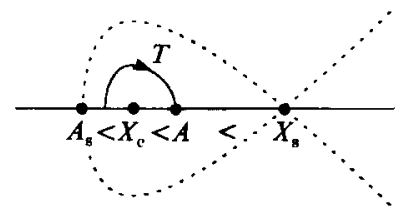

(b)

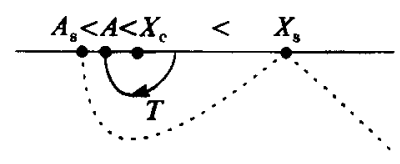

(d)

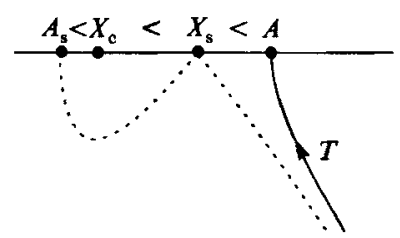

Figure 12. $(a-d)$. Schematic diagrams showing how the nature of an upstream trajectory $T$ is dependent on the relationship between $A$, the value of $\alpha$ when it meets the $X$-axis, and $A_{\mathrm{s}}, X_{\mathrm{c}}$ (the position of a centre) and $X_{5}$ (a saddle point). 


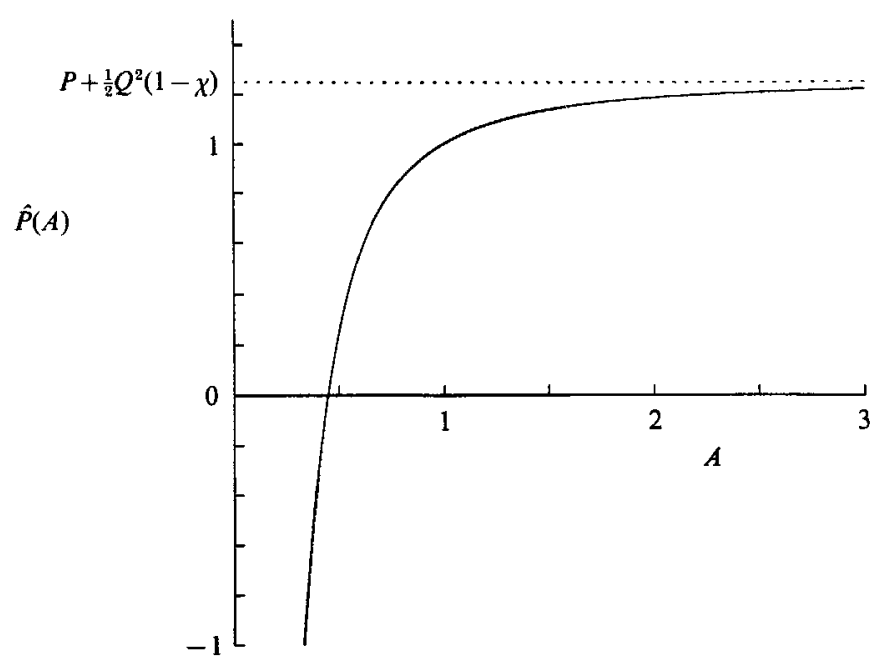

Figure 13. The function $\hat{P}(A)$ plotted against $A$ for $Q=P=1, \chi=0.5$.

trajectories representing a narrowing tube.) In addition to these, if $X_{\mathrm{c}}(Q, \hat{P}(A))=A$ or $X_{\mathrm{s}}(Q, \hat{P}(A))=A$ then $T$ will be a centre $C_{\mathrm{U}}$ or a saddle $S_{\mathrm{U}}$, and if

$$
A=A_{\mathrm{s}}(Q, \hat{P}(A))
$$

then $T$ is an upstream saddle-point trajectory on the $(X, Y)$-phase plane such as that through $(\bar{A}, 0)$ in the example given earlier (see figure $10 a$ ). A solution of $(4.22)$ then satisfies $A=\bar{A}$ (and correspondingly $\bar{X}=X_{\mathrm{s}}(Q, \dot{P}(\bar{A}))$ ).

So for given $(Q, P)$, knowing the behaviour of the functions

$$
X_{\mathrm{s}}(Q, \hat{P}(A))-A, \quad X_{\mathrm{c}}(Q, \hat{P}(A))-A, \quad A_{\mathrm{s}}(Q, \hat{P}(A))-A
$$

with respect to $A$ will reveal the nature of the upstream half of the $\left(\alpha, \alpha_{x}\right)$-phase plane.

$X_{\mathrm{s}}, X_{\mathrm{c}}$ and $A_{\mathrm{s}}$ as functions of $A$ are parameterized by $\hat{P}$ (itself a function of $A$ given by (4.8) and plotted on figure 13) and are continuously defined for $\hat{P}$ between $-\infty$ and $0, \hat{P}^{\dagger}, \hat{P}^{\ddagger}$ or $P+\frac{1}{2}(1-\chi) Q^{2}$ (the asymptote of $\hat{P}$ as $\left.A \rightarrow \infty\right)$, depending on the value of $Q$. In the lower limit $\hat{P} \rightarrow-\infty, A \rightarrow 0$, it is easily shown that $X_{\mathrm{s}}$ and $A_{\mathrm{s}}$ are proportional to $A^{-2}$ and $A^{4}$ respectively, while

$$
X_{\mathrm{c}} \sim \frac{1}{(1-\chi)^{\frac{1}{2}}} A .
$$

Thus $X_{\mathrm{c}}-A$ and $A_{\mathrm{s}}-A$ both pass through zero when $A=0$, but with positive and negative gradients respectively. At the upper limit of $\hat{P}, A$ takes a limiting value which we denote by $A^{*}$, which may be finite or infinite. Three different types of behaviour may be described, as summarized on figure 14 .

Type $1.0<P<P^{A}$ for $0<Q<(3 / 2)^{\frac{1}{2}}$, and $0<P<P^{C}(Q)$ for $(k / \chi)^{\frac{1}{2}}<Q$, where

$$
\begin{aligned}
& P^{A}(Q)=-f\left(\chi Q^{2} ;\left(\frac{2}{3} Q^{2}\right)^{2}\right), \\
& P^{C}(Q)=-f\left(\chi Q^{2} ;\left(\frac{Q^{2}}{k}\right)^{\frac{1}{3}}\right)
\end{aligned}
$$

$P=P^{A}(Q)$ and $P=P^{C}(Q)$ are plotted as a functions of $Q$ on figure $14(a)$ (and also on figure 11). Note that $X_{\mathrm{c}}-A=0$ at $A=C_{\mathrm{U}}$ and $X_{\mathrm{s}}-A=0$ at $A=S_{\mathrm{U}}$; these will be 


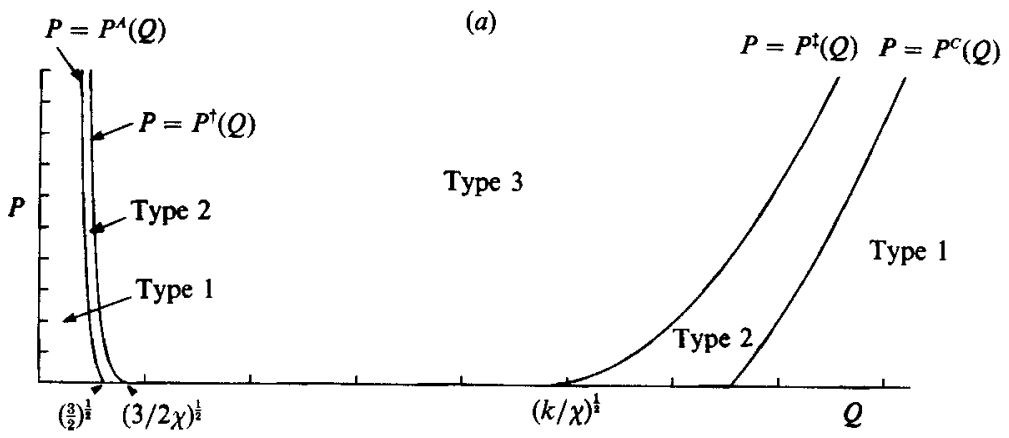

(b) Type 1
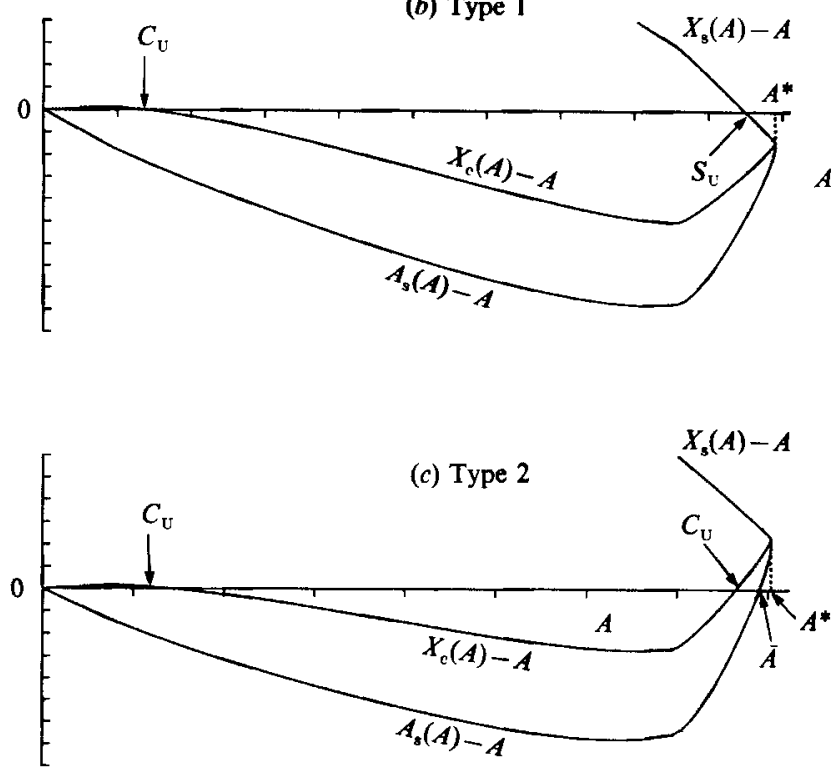

(d) Type 3

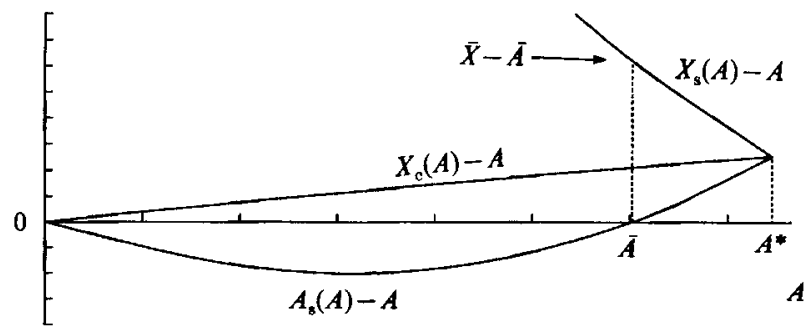

Figure 14. Three types of behaviour of the functions $A_{\mathrm{s}}(A)-A, X_{\mathrm{o}}(A)-A$ and $X_{\mathrm{s}}(A)-A$ : in $(a)$ the regions of $(Q, P)$-space corresponding to each type are shown; $(b),(c)$ and $(d)$ are graphs of the functions for parameter values in each region.

coincident with the downstream singularities $C_{\mathrm{D}}$ and $S_{\mathrm{D}}$ which exist for these parameter values in the $(X, Y)$-phase plane.

Type 2. $P^{A}(Q)<P<P^{\dagger}(Q)$ for $Q<(3 / 2)^{\frac{1}{2}}, 0<P<P^{\dagger}(Q)$ for $(3 / 2)^{\frac{1}{2}}<Q<(3 / 2 \chi)^{\frac{1}{2}}$, and $\max \left(0, P^{C}\right)<P<P^{\ddagger}(Q)$ for $(k / \chi)^{\frac{1}{2}}<Q$. In this parameter regime the downstream singularities $C_{\mathrm{D}}$ and $S_{\mathrm{D}}$ are matched by two upstream centres $C_{\mathrm{U}}$. Note also 
that an upstream saddle point exists which always lies to the right of these singularities. For any $(Q, P)$ on either curve $P=P^{\dagger}(Q)$ or $P=P^{\ddagger}(Q)$, the two roots $C_{\mathrm{U}}$ of $X_{\mathrm{c}}-A=0$ coalesce to form a double root, and this point corresponds to the degenerate downstream singularity $(C S)_{\mathrm{D}}$ that exists for these parameter values.

Type 3. $P^{\dagger}(Q)<P$ for $Q<(3 / 2 \chi)^{\frac{1}{2}}, 0<P$ for $(3 / 2 \chi)^{\frac{1}{2}}<Q<(k / \chi)^{\frac{1}{2}}$, and $P^{\ddagger}(Q)<P$ for $(k / \chi)^{\frac{1}{2}}<Q$. An upstream saddle-point trajectory $\bar{A}$ always exists. There are no singularities otherwise. Along the curve $P=\hat{P}^{B}(Q)$ (see (3.25) and figure $11 \mathrm{ii}$ ), it is easily shown that $\bar{A}=1$. To its left $\bar{A}<1$, and for points to its right $\bar{A}>1$.

Finally, we may determine for which parameter values the upstream saddle point $\bar{X}$ corresponding to $\bar{A}$ takes the value 1 . First, it may easily be shown that if $Q<(3 / 2)^{\frac{1}{2}}$, then

$$
\begin{aligned}
A_{\mathrm{s}}(Q, 0) & =\left[\left(1-\frac{1}{2} Q^{2}\right)^{-\frac{1}{2}}-1\right]^{2} \\
& =A_{0}(Q),
\end{aligned}
$$

say (see figure $5 a$ ). Then we have $\bar{X}=1$ if $\hat{P}(\bar{A})=0$ and $\bar{A}=A_{0}$; these conditions may be combined to give

$$
\begin{aligned}
P & =\frac{1}{2}(1-\chi) Q^{2}\left(\frac{1}{A_{0}^{2}}-1\right) \\
& =P^{0}(Q),
\end{aligned}
$$

say. The curve $P=P^{0}(Q)$ is shown on figure $11(\mathrm{i})$. If $Q<(3 / 2)^{\frac{1}{2}}$ and $P^{A}(Q)<P<P^{0}(Q)$ then $\bar{X}<1$; for all larger $P$ or larger $Q$ such that it exists, $\bar{X}>1$.

\subsection{The nature of the solutions}

We start by examining the five regions of the $(Q, P)$-plane bounded by the curves $P=P^{\dagger}(Q), P=P^{A}(Q)$ and $P=P^{0}(Q)$ (shown on figure $11 \mathrm{i}$ ), and so for the moment assume that $Q<k^{\frac{1}{2}}$. We may summarize the relevant parts of $\$ 4.2$ as follows.

(i) Above $P=P^{\dagger}(Q)$, there are no downstream singularities. Beneath it we have a downstream centre $C_{\mathrm{D}}$ and a saddle point $S_{\mathrm{D}}$ in $0<X<1$.

(ii) Beneath $P=P^{A}(Q)$ there is an upstream centre $C_{\mathrm{U}}$ at $C_{\mathrm{D}}$ and an upstream saddle point $S_{\mathrm{U}}$ at $S_{\mathrm{D}}$. Above it, but beneath $P=P^{\dagger}(Q), C_{\mathrm{U}}$ and $C_{\mathrm{D}}$ are still coincident but another $C_{\mathrm{U}}$ is found at $S_{\mathrm{D}}$.

(iii) For all $(Q, P)$ above $P=P^{A}(Q)$ there exists a saddle-point trajectory through $(\bar{A}, 0)$ (where $\bar{A}$ lies to the right of any singularities that may exist), which meets the $X$-axis at an upstream saddle point $(\bar{X}, 0)$. Beneath $P=P^{0}(Q), \bar{X}<1$ and above it $\bar{X}>1$.

Labelling the five regions of the $(Q, P)$-plane as shown on figure 11 (i), we may now look for solutions of (4.14).

(a) $Q<(3 / 2)^{\frac{1}{2}}, P<P^{A}(Q)$

A typical phase portrait is shown on figure $15(a)(\mathrm{i})$. We have $C_{\mathrm{U}}$ at $C_{\mathrm{D}}$ and $S_{\mathrm{U}}$ at $S_{\mathrm{D}}$. No upstream saddle-point trajectory $(\bar{A})$ exists. (We see from figure $14(b)$ that for these 'Type 1' parameter values, if $A<C_{\mathrm{U}}$ then $A_{\mathrm{s}}(A)<A<X_{\mathrm{c}}(A)$, so the corresponding upstream trajectories are of the form shown in figure $12 b$ ). The upstream and downstream boundary conditions (4.5) can only be satisfied for trajectories that pass through $S_{\mathrm{D}}<A \leqslant 1 . I(A) \rightarrow \infty$ as $A \rightarrow S_{\mathrm{D}}+$, as shown in figure $15(a)$ (ii). Solutions exist for all lengths of tube with the tube everywhere collapsed.

(b) $Q<(3 / 2)^{\frac{1}{2}}, P^{A}(Q)<P<\min \left(P^{\dagger}(Q), P^{0}(Q)\right)$

Corresponding to $C_{\mathrm{D}}$ and $S_{\mathrm{D}}$ are two upstream centres $C_{\mathrm{U}}$, and to the right of $S_{\mathrm{D}}$ there is an upstream saddle-point trajectory through $(\bar{A}, 0)$ which meets the $X$-axis at 
$(\bar{X}, 0)$ with $\bar{X}<1$, as shown in figure $15(b)(i) . I(A)$ is only defined for $\bar{A}<A \leqslant 1$ (see figure $15 b \mathrm{ii}$ ), and is similar in form to case $(a)$.

(c) $Q<Q^{*}, P^{\dagger}(Q)<P<P^{0}(Q)$ where $P^{\dagger}\left(Q^{*}\right)=P^{0}\left(Q^{*}\right)$

There are no singularities on the phase plane, but we have a saddle-point trajectory through $(\bar{A}, 0)$ and $(\bar{X}, 0)$, where $\bar{X}<1$ (see figure 15ci). All upstream trajectories in $A<\bar{A}$ form loops that do not cross $X=1$, so $I(A)$ is defined only for $\bar{A}<A \leqslant 1$, as in case $(b)$, and as shown in figure $15(c)$ (ii).

(d) $Q^{*}<Q<(3 / 2)^{\frac{1}{2}}, P^{0}(Q)<P<P^{\dagger}(Q)$ and $(3 / 2)^{\frac{1}{2}}<Q<(3 / 2 \chi)^{\frac{1}{2}}, 0<P<P^{\dagger}(Q)$ Since $P<P^{\dagger}(Q), C_{\mathrm{D}}$ and $S_{\mathrm{D}}$ are present again, matched by two upstream centres as in ease $(b)$, so the downstream half-plane is just as in figure 15(b) (i) and no upstream trajectories through $(A, 0)$, where $A<S_{\mathrm{D}}$, can satisfy (4.5a). Similarly, an upstream saddle-point trajectory through $(\bar{A}, 0)$ and $(\bar{X}, 0)$ exists, with $S_{\mathrm{D}}<\bar{A}<1<\bar{X}$. Between $S_{\mathrm{D}}$ and $\bar{A}$ there is an upstream trajectory through $\left(A_{1}, 0\right)$ which loops round to $(1,0)$, just as in the example shown in figure $10(a)$, and likewise no upstream trajectory through $(A, 0)$, with $S_{\mathrm{D}}<A<A_{1}$, ever meets $\alpha=1$. Thus $I(A)$ is just as in figure $10(c)$; it is defined only for $A_{1} \leqslant A \leqslant 1$, representing tubes that are everywhere collapsed (LU), and it has a branch (ULU) defined for $A_{1} \leqslant A<\bar{A}$ representing tubes that are swollen just downstream of $x=0$ and constricted further downstream.

(e) $0<Q<(3 / 2 \chi)^{\frac{1}{2}}, \quad \max \left(P^{\dagger}(Q), \quad P^{0}(Q)\right)<P$ and $(3 / 2 \chi)^{\frac{1}{2}}<Q<k^{\frac{1}{2}}, \quad 0<P$ and $k^{\frac{1}{2}}<Q, \hat{P}^{B}(Q)<P$

Phase planes for these parameter values are just as presented in figure $10(a) ; I(A)$ (see figure $10 c$ ) is defined only for $A_{1}<A<1$.

We now turn to the remainder of the $(Q, P)$-plane, where $Q>k^{\frac{1}{2}}$ (see figure $11 \mathrm{ii}$ ). From $\$ 4.2$, the phase plane has the following properties, much as before.

(i) Above $P=P^{\ddagger}(Q)$, there are no downstream singularities. Beneath it, a centre $C_{\mathrm{D}}$ and a saddle point $S_{\mathrm{D}}$ are found in $X>1$.

(ii) Beneath $P=P^{C}(Q)$ there is an upstream centre $C_{\mathrm{U}}$ at $C_{\mathrm{D}}$ and a saddle point $S_{\mathrm{U}}$ at $S_{\mathrm{D}}$. Between $P=P^{C}(Q)$ and $P=P^{\ddagger}(Q)$, the upstream singularity at $S_{\mathrm{D}}$ is a centre.

(iii) Everywhere above $P=P^{C}(Q)$ an upstream saddle-point trajectory through $(\bar{A}, 0)$ exists. Beneath $P=P^{\ddagger}(Q), \bar{A}$ lies to the right of $S_{\mathrm{D}}$; between $P=\hat{P}^{B}(Q)$ and $P=P^{\ddagger}(Q), \bar{A}>1$, but above $\hat{P}^{B}(Q), \bar{A}<1$.

There are three regions of the $(Q, P)$-plane that remain to be discussed, all lying to the right of $P=\hat{P}^{B}(Q)$ on figure 11 (ii).

(f) $k^{\frac{1}{2}}<Q, P<\hat{P}^{B}(Q)$ and $P^{\ddagger}(Q)<P$ when $(k / \chi)^{\frac{1}{2}}<Q$

No downstream singularities are present but an upstream saddle-point trajectory through $(\bar{A}, 0)$ exists, where $\bar{A}>1$ (see figure $15 d \mathrm{i}$ ). $I(A)$ is only defined for $A_{1} \leqslant A \leqslant 1$, where the upstream trajectory through $\left(A_{1}, 0\right)$ passes through $(1,0)$. As in case $(e), I$ has two branches (shown as solid curves on figure $15 d \mathrm{ii}$ ) representing tubes which are either everywhere collapsed (LU), or which have a bulge as well as a constriction (ULU). Note that in this case there is an upper limit $I\left(B_{1}\right)$, (where $B_{1}>1$ is the point of intersection of the upstream trajectory through $\left.(1,0)\right)$, to the value of $\lambda$ for which solutions that are at some point constricted exist. However, for parameter values in this range the fully attached solutions that we obtained in $\S 3$ with the tube dilated along its length also exist (assuming that there is negligible 

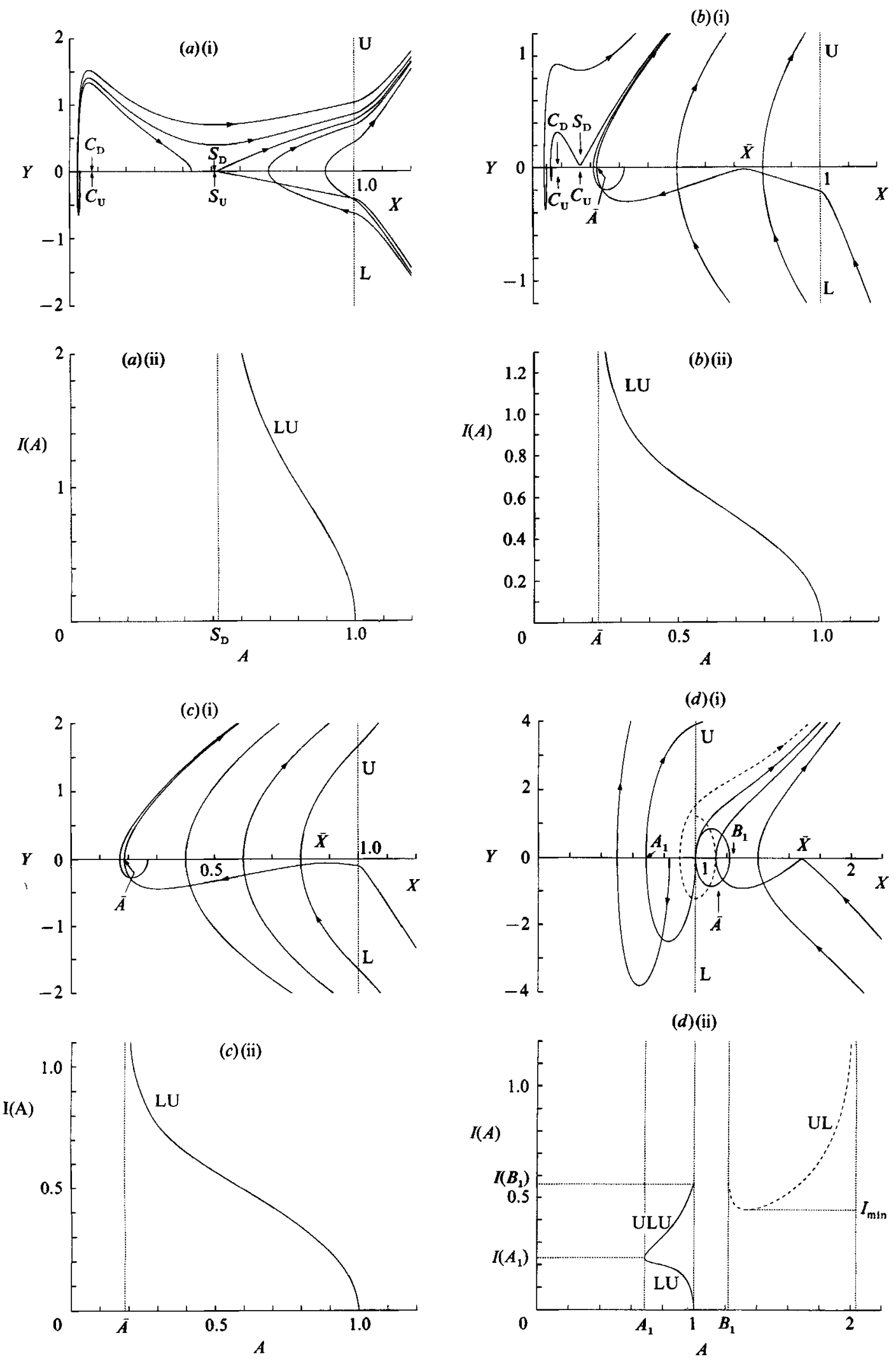

Figure $15(a)(\mathrm{i}, \mathrm{ii}),(b)(\mathrm{i}, \mathrm{ii}),(c)(\mathrm{i}, \mathrm{ii}),(d)(\mathrm{i}, \mathrm{ii})$. For caption see facing page. 

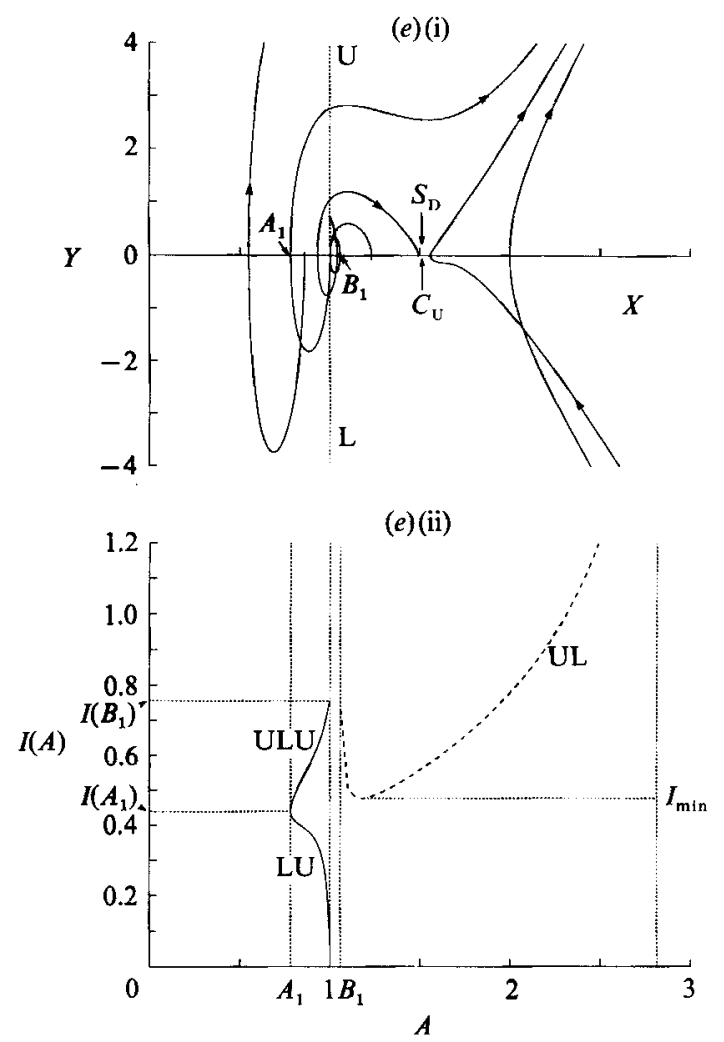

Figure 15. (i) Trajectories in the $\left(\alpha, \alpha_{x}\right)$ phase plane in the separated-flow case; (ii) the corresponding graphs of $I(A)$. Parameter values are: $(a) Q=1, P=1$ (region $a$ of figure 11); (b) $Q=1, P=5$ (region $b) ;(c) Q=1, P=7$ (region $c) ;(d) Q=12, P=8($ region $f) ;(e) Q=14$, $P=4($ region $g) ; k=45$ and $\chi=0.5$ in all cases.

separation at $x=0$ ). We show the (dotted) UL branch of $I$ representing these solutions in figure $15(d)$ (ii), corresponding to the UL branch in figure $6(e)$ (ii) - see figure $6(e)$ (i) for the appropriate trajectories; as before, these dilated solutions exist provided $\lambda>I_{\min }$.

(g) $(k / \chi)^{\frac{1}{2}}<Q, P<P^{\ddagger}(Q)$ and $P>\max \left(0, P^{C}(Q)\right)$

The downstream centre $C_{\mathrm{D}}$ and saddle point $S_{\mathrm{D}}$ are matched by two upstream centres. An upstream saddle-point trajectory lies to the right of $S_{\mathrm{D}}$ in $X>1$, so as far as solutions satisfying (4.5) are concerned, the behaviour is just as in case $(f)$. See figures $15(e)(\mathrm{i})$ and (ii).

(h) $Q^{C}<Q, P<P^{C}(Q)$, where $P^{C}\left(Q^{C}\right)=0$

The only difference between this case and case $(g)$ is that $S_{\mathrm{D}}$ is now matched by an upstream saddle point $S_{\mathrm{U}}$, but this has no effect on the behaviour of $I$ which is the same as that shown in figure $15(e)$ (ii).

Finally, mention must be made of the case $P<0$. When there is no separation, it was found in $\S 3$ that, as well as the expected distended solution, there exists a surprising solution with the tube collapsed at some point (see the curve LU in figure 6aii). When separation is included in the calculation, the distended solution is obviously unaffected but the collapsed one vanishes. This is because all upstream 


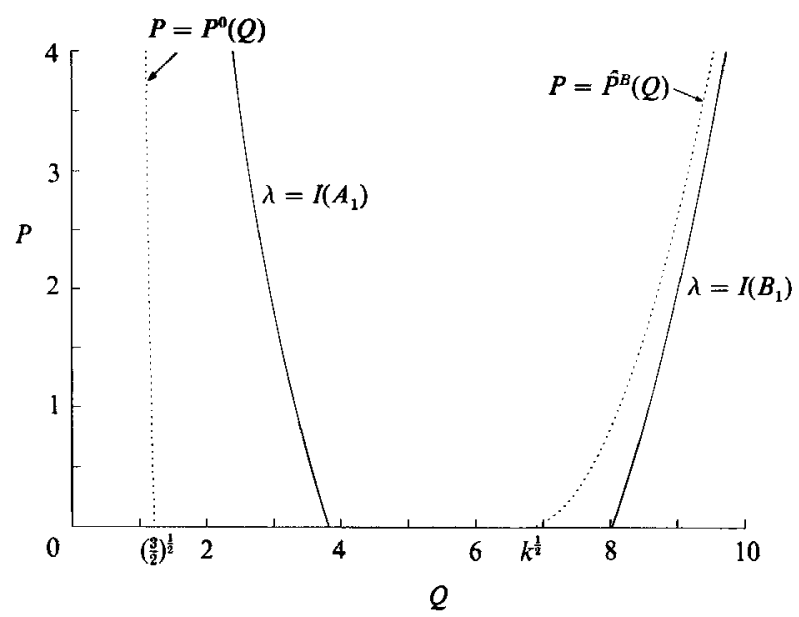

Figure 16. The curves $\lambda=I\left(A_{1}\right)$ and $\lambda=I\left(B_{1}\right)$ are plotted for $\lambda=1$. A solution with the flow separated downstream of a constriction exists for all $(Q, P)$ to the left of $\lambda=I\left(B_{1}\right)$.

trajectories in $A \leqslant A_{1}$ bend over to meet the $X$-axis in $X<1$, making it impossible to satisfy the upstream boundary condition $(4.5 a)$.

\subsection{Conclusions}

We may draw the following conclusions. Beneath the curve $P=P^{0}(Q)$ in the $(Q, P)$ plane, (regions $a, b$ and $c$ on figure 11), for all lengths of tube and tensions there exists a steady solution with the tube held open at both ends but at every intermediate point having $\alpha<1$, with the flow separating downstream of the point of greatest constriction. Between $P=P^{0}(Q)$ and $P=\hat{P}^{B}(Q)$, (regions $d$ and $e$ ), such solutions for a tube of dimensionless length $\lambda$ exist only beneath the contour $\lambda=I\left(A_{1}\right)$, where $A_{1}=A_{1}(Q, P)$. Such a contour is shown in figure 16 . For longer tubes, steady solutions are found which have a swelling just downstream of the opening of the tube as well as a constriction further downstream, at which separation takes place.

In the remaining regions of $(Q, P)$-space $(f, g$ and $h)$, however, solutions for which separation occurs exist only for sufficiently short tubes. Again two types of configuration are then possible: for very short tubes (i.e. for $\left.\lambda<I\left(A_{1}\right)\right), \alpha \leqslant 1$ everywhere between the end points; for longer tubes with $I\left(A_{1}\right)<\lambda<I\left(B_{1}\right)$, there is a bulge in the tube with a constriction further downstream. With $\lambda>I\left(B_{1}\right)$, for these parameter values a unique solution always exists in which the tube is dilated everywhere, so that no separation occurs.

\section{Results}

\subsection{Fully attached flow}

For a given dimensionless tube length $\lambda$, the regions of $(Q, P)$ parameter space for which solutions to

$$
\lambda=I(A ; Q, P)
$$

exist are as shown on figure 8 . Beneath $\lambda=I_{\max }(Q, P)$, two solutions exist with the elastic tube everywhere collapsed. In some circumstances (e.g. for subcritical entry flow and small pressures - see figure 6 bii) these two solutions may be explicitly associated with sub- and supercritical flows: the larger value of $A$ corresponds to flow that is everywhere subcritical; the other root is small enough for the flow to be 
supercritical at the constriction. For increasing pressures and flow rates, the two roots gradually approach one another, the 'subcritical' one becoming smaller and the 'supercritical' one larger, until eventually the contour $\lambda=I_{\max }$ is reached; the roots are then equal and the tube may be said to be on the point of choking. There is no simple relationship between the flow velocity $u$ and the wave speed $c$ at this point because of the dispersive effect of longitudinal tension. Indeed, the dimensional tube length and the degree of tension, which are both incorporated in the parameter $\lambda$, influence the pressures and flow rates at which choking will occur.

Between $\lambda=I_{\max }$ and $\lambda=I_{\min }$ (also plotted on figure 8), no solutions exist. However for all $(Q, P)$ to the right of $\lambda=I_{\min }$, the tube may take up a configuration in which it is dilated everywhere; for sufficiently large $Q$ a variety of periodic solutions are also possible, with the collapsible segment alternately dilated and contracted along its length. Such configurations, which are also obtained when $P<0$, were discussed in greater detail by Reyn (1987).

\subsection{Separated flow}

Whereas in figure 8 we see a region of $(Q, P)$-space for which no solutions exist, the corresponding graph (figure 16) shows that solutions with either separated flow downstream of a constriction or a dilated configuration may be found for all $Q, P>0$. However, only one fully collapsed solution for $(Q, P)$ beneath $\lambda=I\left(A_{1}\right)$ is found (the 'supercritical' solution of fully attached flow is never obtained). Correspondingly, no choking phenomenon occurs. Rather, as flow rate increases a bulge develops in the tube upstream of the constriction (see figure $17 a, b$ ), so that to the right of $\lambda=I\left(A_{1}\right)$ the tube is both dilated and contracted (figure 17c). Further increases in flow rate enlarge the bulge and force open the constriction until, along $\lambda=I\left(B_{1}\right)$, the tube is fully dilated and no energy loss by separation occurs. For larger flow rates, there is a solution with the flow fully attached and the tube becoming increasingly dilated, but the transition to this solution as we cross $\lambda=I\left(B_{1}\right)$ is not achieved continuously (as can be seen from figure 15eii, say).

\subsection{Comparison with experiment}

Whenever a solution of (5.1) exists with separation taking place downstream of a constriction, it is possible to calculate the pressure drop down the collapsible segment using (4.9) and thus to compare it with the corresponding flow rate.

There are two ways of presenting this relationship which have been favoured by experimentalists, and which can easily be reproduced by this model. The first approach is that taken by Brower \& Scholten (1975), Bonis \& Ribreau (1978) and also by Bertram (1986): with $p_{\mathrm{e}}-p_{2}$ held constant, $p_{1}-p_{2}$ is plotted against $Q$. Bertram's experimental curves are reproduced in figure $18(a)$. Since he used a thickwalled tube for these measurements, good quantitative agreement between our (thinwalled) model and his results cannot be anticipated. It should also be remembered that $p_{1}$ and $p_{2}$ are not measured precisely at the ends of the collapsible segment, but at short distances up- and downstream of these points.

Figure 18(b) shows curves calculated for five different values of $p_{\mathrm{e}}-p_{2}$, with $\lambda$ and $\chi$ held constant at values of 25.2 and 0.2 respectively. The left-hand part of the curves, corresponding to tubes collapsed along their length (where $p_{1}-p_{2}<p_{\mathrm{e}}-p_{2}$ on figure $18 b$ ) show an increasing resistance to flow as $Q$ increases, but not in so linear or so rapid a manner as obtained experimentally, presumably a reflection of our neglect of friction. For each curve the rate of increase drops just before the pressure drop reaches the corresponding value of $p_{\mathrm{e}}-p_{2}$, as the collapsed region is forced 

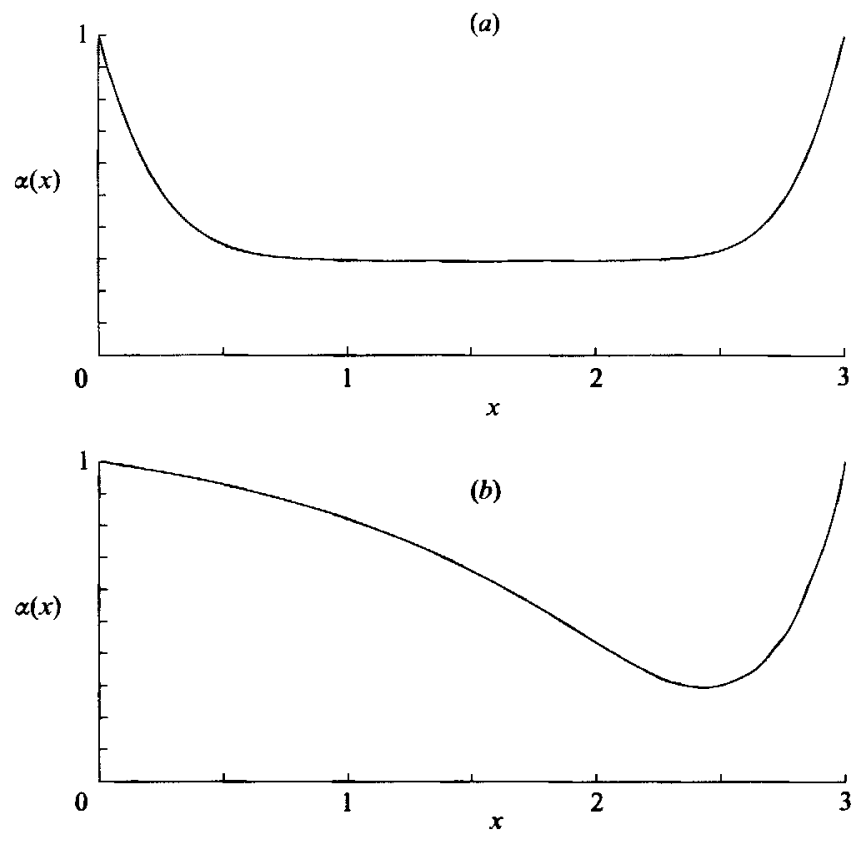

(c)

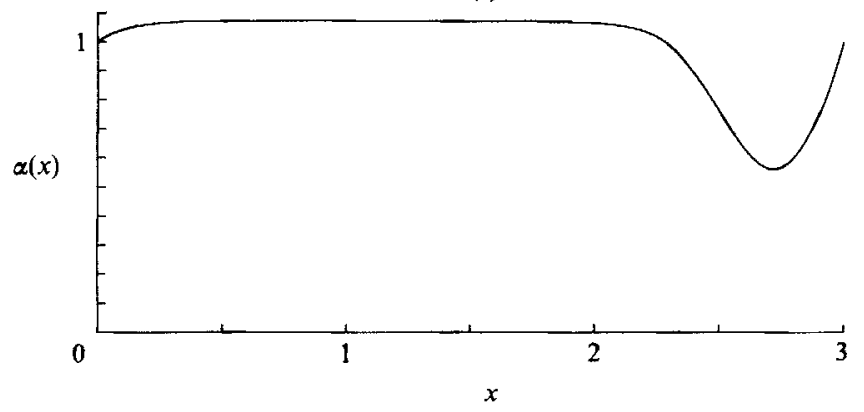

Fraure 17. Three tube shapes calculated for $\lambda=3, \chi=0.2, k=45$ and $P=5$, with the flow rate increasing through values $(a) Q=0.7$, (b) $Q=2.3$, (c) $Q=5.0$, showing how a bulge develops upstream while the flow separates at the constriction downstream.

open; our model predicts upstream swelling of the tube generally just beyond this bend in the curve, when $p_{1}$ is very slightly greater than $p_{\mathrm{e}}$. Bertram measured similar bends in the curves, but always when $p_{1}-p_{2}$ was a little less than $p_{\mathrm{e}}-p_{2}$. For all but the smallest downstream transmural pressures (when there is little collapse and friction is probably the predominant cause of pressure drop), he found it impossible to obtain steady flows beyond this bend. This suggests that there may be a connection between the loss of stability and the swelling predicted by our model.

In general, solutions of (5.1) are all extremely close to the asymptotes of $I$ (such as $S_{\mathrm{D}}$ or $\bar{A}$ ), and the pressure-drop curves are largely indistinguishable for all values of $\lambda \geqslant 5$, say, and the values of $\lambda$ corresponding to Bertram's experiments are greater than 5 (see $\$ 2.6$ ). (The jet energy loss only occurs in the far downstream (constricted) section of the tube, as in figure $17(c)$, and we are neglecting a viscous contribution to the pressure drop which grows as $\lambda$ increases.) Thus once $p_{1}>p_{\mathrm{e}}$ in figure $18(b)$, a good approximation for the pressure drop is given by (4.9) but with $A$ replaced by 

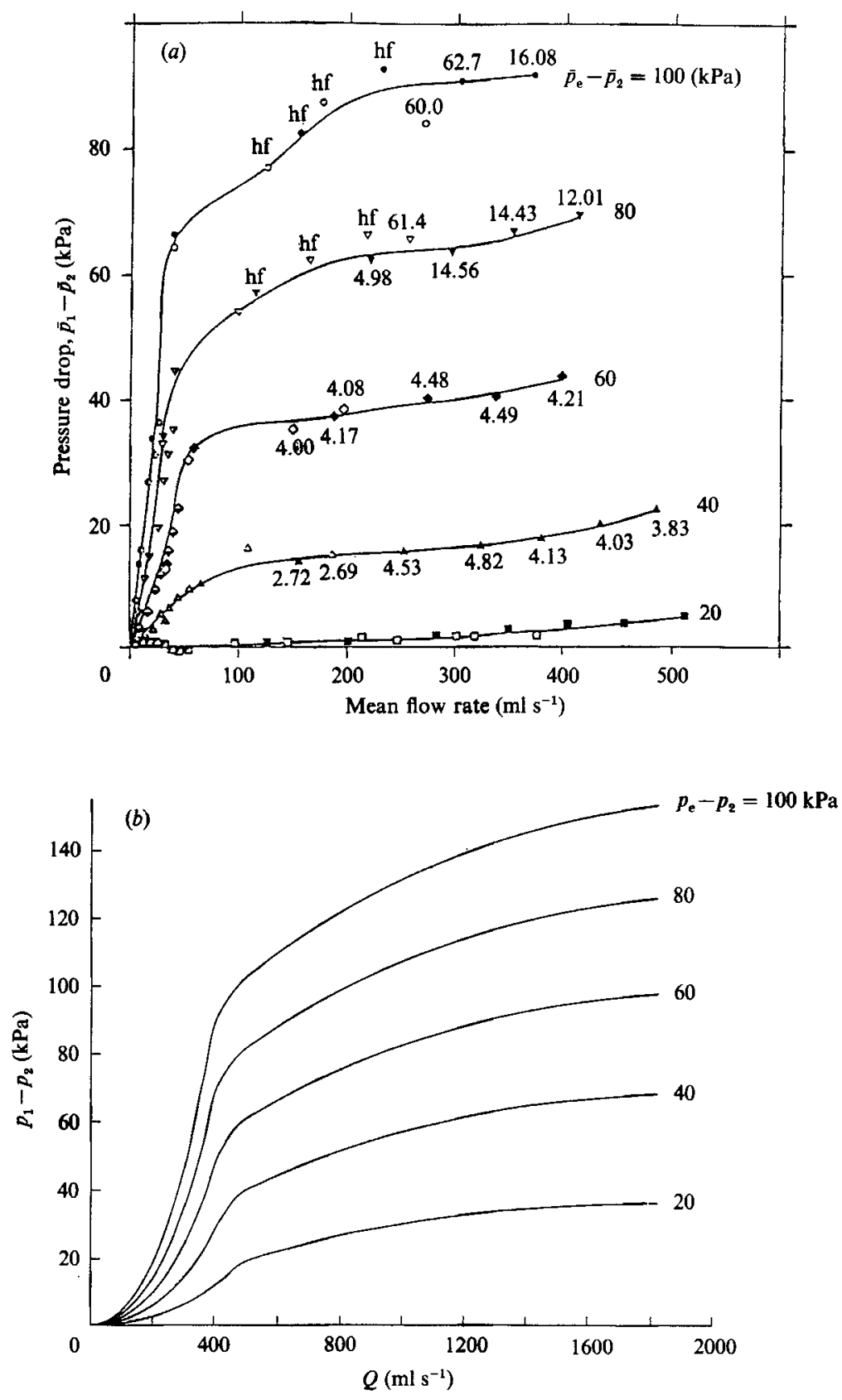

Figure 18. Comparison between (a) experimental measurements by Bertram (1986) of pressure drop $p_{1}-p_{2}$ down the collapsible tube plotted against flow rate (reproduced with permission), for fixed values of $p_{\mathrm{e}}-p_{2}$; and $(b)$ curves calculated over a wider range of flow rates but otherwise using his parameter values: $\lambda=25.2, k=45,0<Q<4$ and $P=1.8,3.6,5.4,7.2$ and $9.0 ; \chi$ is chosen to be 0.2 . The different symbols used in $(a)$ to mark the measured points correspond to different downstream resistances used in the experiment, while the adjacent numbers refer to the frequency of observed oscillations, and for these cases time-averaged pressures are recorded. 
$\bar{A}(Q, P)$, the limiting size of the constriction when the tube has a bulge upstream. For fixed $p_{\mathrm{e}}-p_{2}$, as $Q$ rises the bulge widens but $\bar{A}$ decreases, causing an increase in resistance to the flow. However, there is a point (beyond the range of $Q$ chosen for figure $18 b$ ), beyond which further rises in $Q$ force the tube to widen everywhere, so that the constriction diminishes until finally (when $Q$ and $P$ satisfy $P=\hat{P}^{B}(Q)$ ) the tube is everywhere dilated, and there is no separation, no energy loss and zero pressure drop. For such large flow rates frictional pressure loss would of course be substantial.

Our predictions are given in dimensional form, and it is clear that there is reasonable quantitative agreement between the pressure values of figures $18(a)$ and (b): the 'corners' in each calculated curve occur almost exactly where $p_{1}=p_{\mathrm{e}}$, whereas Bertram never measured $p_{1}>p_{\mathrm{e}}$, so we overestimate the pressure drop slightly. (Better agreement is obtained with $\chi$ small, 0.2 say, rather than 0.5 , as the smaller $\chi$ is, the lower is the maximum pressure drop. This is counter to expectation - one would expect an increase in $\chi$ to cause a reduction in pressure drop, as more pressure recovery is then allowed along the jet emerging from the constriction, but this is only observed for small flow rates when the tube is everywhere collapsed.) The flow rate scales do not agree well, however: the whole of figure $18(a)$ is modelled by the left-hand part of $18(b)$, with $0<Q<500 \mathrm{ml} \mathrm{s}^{-1}$, and so the predicted $p_{1}-p_{2}$ vs. $Q$ curve has a much gentler slope in the predictions than in the experiments.

Agreement with the experiments of Bonis \& Ribreau (1978) is more encouraging, which is more understandable since they used a thinner-walled tube than Bertram. On their plot of the pressure-flow rate relations (figure 19a) they mark three regimes, bounded by the curves marked $p_{1}=p_{\mathrm{c}}$ and $p_{1}=p_{\mathrm{e}}$. The former describes the points where the opposite walls of the tube just come into contact. In the region to the left of this curve, they report that the tube is collapsed along its entire length (e.g. figure $17 a$ ), with the flow passing down the parallel lobes within the tube. It is in this region that frictional energy loss predominates over jet energy loss, and accordingly our theory (using exactly their parameter values, and $\chi=0.2$ ) severely underestimates the measured pressure drop. In the region adjacent to this, Bonis \& Ribreau report the upstream half of the tube opening up more than the downstream half as the flow rate increases, which corresponds with our calculations (see figure $17 \mathrm{~b})$. The boundary of the third region, on which $\hat{P}=0$, we mark on our diagram (figure 19b) with a dotted curve. Along this line the curvature of the tube wall at the upstream end, $\frac{1}{2} \alpha_{x x}(0)$, changes sign and for only slightly larger flow rates the tubc is dilated in its upstream half and constricted further downstream, as in figure $17(c)$; correspondingly, Bonis \& Ribreau describe the tube as open for its entire length, except for a neck at the downstream end. The pressure drop-flow rate curves in this third region are in excellent agreement (justifying our neglect of friction in this case) showing only weak resistance to flow. But this may be fortuitous: the tube shape when $Q=27 \mathrm{~cm}^{3} / \mathrm{s}, P=30 \mathrm{cmH}_{2} \mathrm{O}$ has the 'neck' in the tube restricted to the final $15 \%$ of its length, with the area changing from $\alpha=0.1$ to $\alpha=1$ over half this distance. Thus for these parameter values our assumption that area variations occur over long lengthscales is not justified, and the consistency of our results must be called into question. Unsteady behaviour is reported by Bonis \& Ribreau to occur shortly after the 'corner' in the curves, corresponding to Bertram's observations, and in this regime therefore they plot time-averaged quantities.

An alternative method of plotting pressure drop against flow rate is to fix the resistance in the downstream rigid tube, $\eta_{2}$, and the external pressure, $p_{\mathrm{e}}$, so that as $Q$ increases $p_{\mathrm{e}}-p_{2}=p_{\mathrm{e}}-\eta_{2} Q^{2}$ decreases. In this case we seek to compare the 

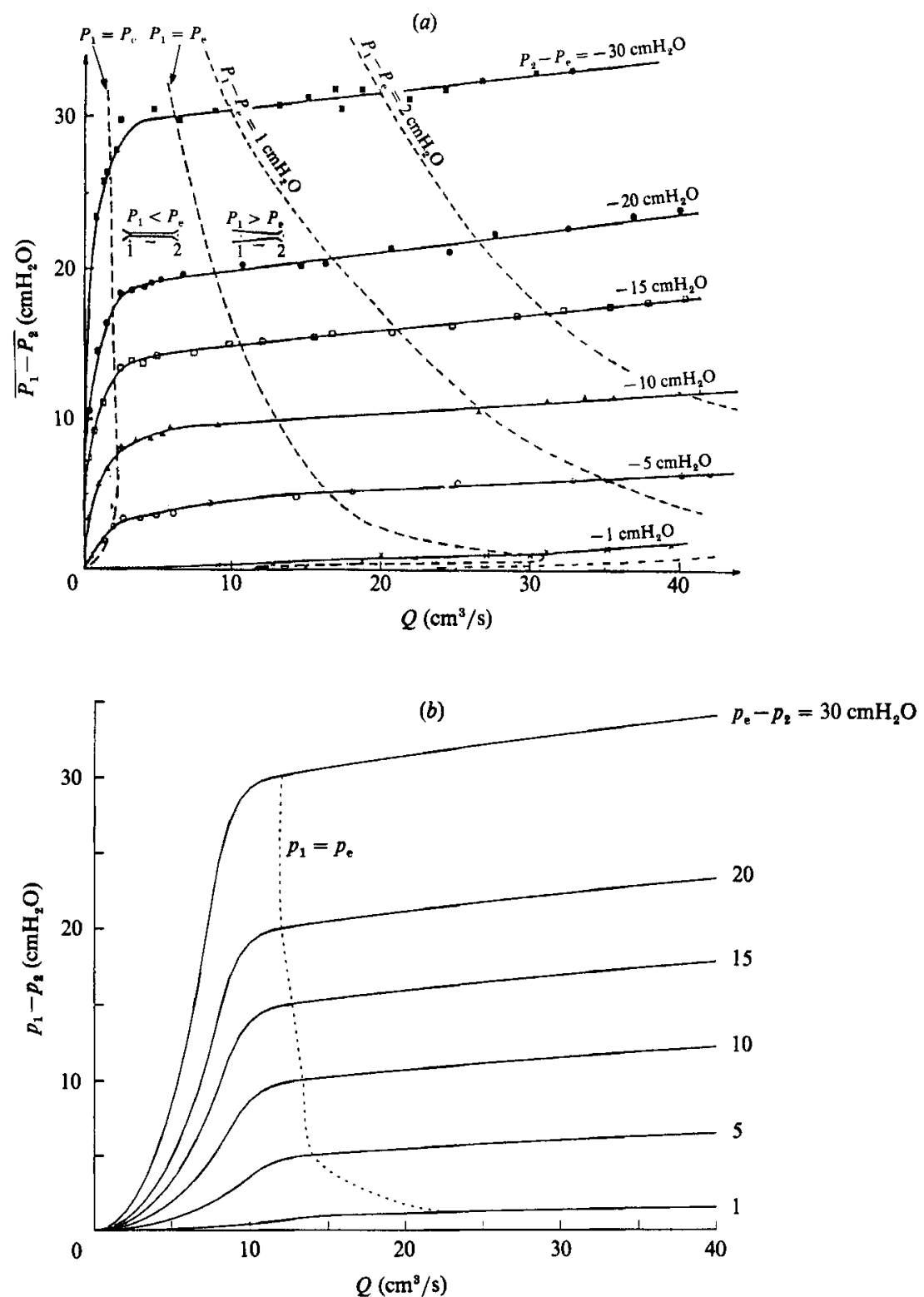

Figure 19. (a) Curves of pressure drop v8. flow rate for fixed values of $p_{\mathrm{e}}-p_{2}$, measured by Bonis \& Ribreau (1979) (reproduced with permission); and $(b)$ the same quantities calculated using their parameter values: $\lambda=2.9, k=2250,0<Q<2.72, P=5.52 \times(1,5,10,15,20,30)$; again $\chi=0.2$. The points at which the upstream transmural pressure difference is zero $\left(p_{1}=p_{\mathrm{e}}\right)$ is shown as a dotted line in both diagrams.

predictions with Conrad's (1969) experiments, for which parameter values were also given in $\$ 2.6$, and for which some results are given in figure $20(a)$. The value of $\bar{p}_{\mathrm{e}}=29.5 \mathrm{mmHg}$ corresponds to $p_{\mathrm{e}}=71.3$ with $K_{p}=55.8 \mathrm{~Pa}$ (or $p_{\mathrm{e}}=7.13 \times 10^{4}$ with $K_{p}=5.58 \times 10^{-2} \mathrm{~Pa}$, for the smaller wall thickness); $\eta_{2}$ varies between 109 (curve 1 ), 1960 (curve 7) and 8710 (curve 8); $\lambda=1.1$ (or 0.11 ). Predictions for these parameter values are given in figures $20(b)$ and $20(c)$.

For very small flow rates in Conrad's experiments, $p_{\mathrm{e}}-p_{2}$ is large and the tube 

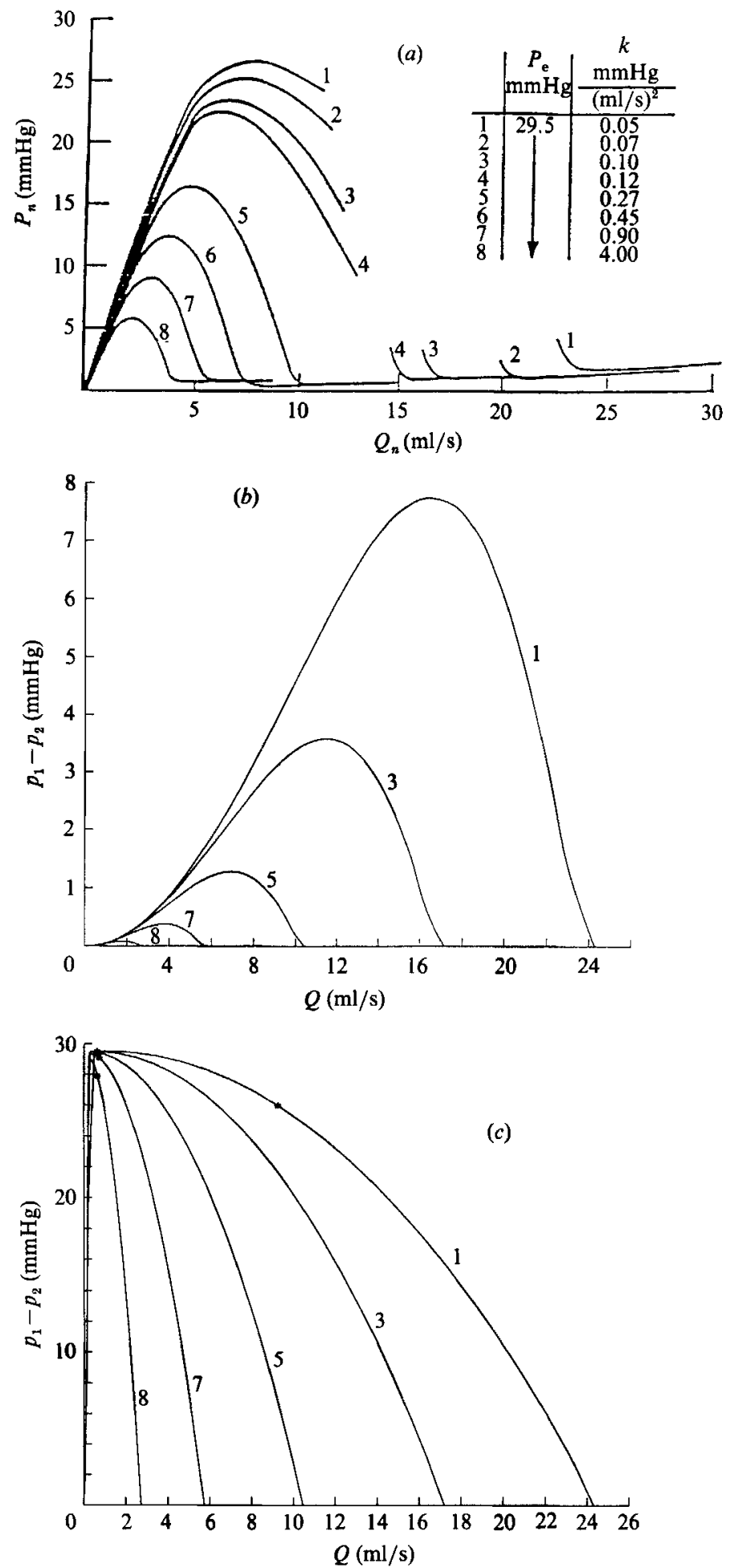

Figure 20. (a) Pressure drop is plotted against flow rate for fixed values of the downstream resistance $\eta_{2}$ by Conrad (1969) (reproduced with permission); the breaks in curves 1-4 correspond to regions of unsteady behaviour. In (b), calculations of pressure drop, for curves $1,3,5,7,8$ using his parameter values are presented: $\lambda=1.1, k=280, p_{\mathrm{e}}=71.3$ and $\eta_{2}=2177 \times(0.05,0.1,0.27,0.9$, 4.0); we take $\chi=0.2$. (c) Similar calculations for parameter values corresponding to a wall thickness of $0.093 \mathrm{~mm}$ instead of $0.93 \mathrm{~mm}\left(\lambda=0.11, k=28000, p_{\mathrm{e}}=7.13 \times 10^{4}\right)$. 
completely collapsed, and pressure drop rises rapidly as $Q$ increases (figure $20 \mathrm{a}$ ). (Note, however, that Conrad measured a more linear variation than we calculated, again demonstrating the importance of friction in these circumstances, both in the collapsible segment and in the downstream rigid tube.) There is a corresponding rise in the upstream pressure $p_{1}$, which proceeds until $p_{1}$ and $p_{\mathrm{e}}$ are approximately equal, at which point the pressure drop has its maximum value. For larger flow rates, $p_{1}>p_{\mathrm{e}}$ and $p_{1}-p_{2}$ falls as $Q$ increases: the rising flow rate and falling downstream transmural pressure force open the constriction so that the tube offers decreasing resistance to the flow. (It is in this parameter range that, for the smaller downstream resistances used in his experiments, Conrad reports unsteady behaviour, shown by the breaks in curves 1-4 in figure $20 a)$. Eventually, when $Q \geqslant\left(p_{\mathrm{e}} / \eta_{2}\right)^{\frac{1}{2}}$, the tube is everywhere dilated and the only mechanism of energy loss is then friction (so that Conrad measured a gentle rise in pressure drop with flow rate, which cannot be reproduced by our model).

We consider first the predictions corresponding to the greater wall thickness of $h=0.93 \mathrm{~mm}$, in figure $20(b)$. The maximum dimensionless flow rate for curve 1 in this figure is 0.8 , so that for all points along the curve the flow rate is small (i.e. it is always subcritical at $x=0$, less than in either Bonis \& Ribreau's or Bertram's experiments). Thus almost everywhere along the curve, $p_{\mathrm{e}}-p_{1}$ is sufficiently large for the tube to be greatly collapsed along its length, and even when $Q$ approaches its maximum value, and $p_{\mathrm{e}}-p_{2}$ becomes quite small, the flow is not great enough for swelling to develop upstream (which Conrad reports in his experiments). Under these conditions, therefore, friction would be a very important cause of pressure drop, and may explain why we underestimate $p_{1}-p_{2}$ by about a factor of four. (With $\chi=0.5$ the difference is even greater, as would be expected for small flow rates.) Another cause of error is that when the elastic segment is greatly collapsed, $\alpha_{x}$ is very large at the joins with the upstream and downstream rigid tubes, so that we fail to satisfy condition (2.12) and we are not justified in neglecting large nonlinear terms when approximating the longitudinal radius of curvature of the tube. However, the shape of the curves in figure $20(b)$ are qualitatively similar to those in figure $20(a)$.

If we consider predictions based on the smaller wall thickness of $0.093 \mathrm{~mm}$, on the other hand (in figure $20 \mathrm{c}$ ), the shape of the curves is completely different, showing an extremely rapid rise in pressure drop at small flow rate, when the tube is highly collapsed, and unrealistically convex curves of decreasing pressure drop as $Q$ increases. These curves look wrong, but in fact that maximum predicted pressure drop is quite well predicted, at least for curve 1. Moreover the tube shape corresponds more closely with experiments, with a bulge in the upstream part of the tube for points to the right of the asterisks in figure $20(c)$, as observed. It is probable that we could achieve acceptable agreement with experiment by selecting a wall thickness somewhere between 0.93 and $0.093 \mathrm{~mm}$, but there is no a priori justification for that.

\subsection{Conclusions}

The shortcomings of this model are numerous, but are not so severe as to prevent quite reasonable qualitative agreement between theory and experiment. Separation of the turbulent jet downstream of a constriction in the tube is shown to have a contribution to total energy loss that is substantial enough for major features of behaviour (such as negative flow resistance in the Conrad experiment) to be predicted. Obviously inclusion of wall friction in the model will be an important refinement; however other improvements, such as a more realistic tube law, may not add greatly to our understanding, at least of steady flow. Of particular interest in the 
dynamical systems context will be the conditions for instability of the steady flows modelled in this paper; a stability analysis is currently underway.

During the course of this work O.E.J. was supported by an SERC Research Studentship. He is additionally grateful for a bursary provided by Smith Associates Ltd.

\section{REFERENCES}

Bertram, C. D. 1982 Two modes of instability in a thick-walled collapsible tube conveying a flow. J. Biomech. 15, 223-224.

Bertram, C. D. 1986 Unstable equilibrium behaviour in collapsible tubes. J. Biomech 19, 61-69.

Bertram, C. D. 1987 The effects of wall thickness, axial strain and end proximity on the pressure-area relation of collapsible tubes. $J$. Biomech $20,863-876$.

Bertram, C. D. \& Pedley, T. J. 1982 A mathematical model of unsteady collapsible tube behaviour. J. Biomech. 15, 39-50.

Bertram, C. D., Raymond, C. J. \& Pedley, T. J. 1989 Mapping of instabilities during flow through collapsed tubes of differing length. J. Fluids Structures (submitted).

Bonis, M. \& Ribreau, C. 1978 Etude de quelques propriétés de l'écoulement dans une conduite collabable La Houille Blanche 3/4, 165-173.

Bonis, M. \& Ribreau, C. 1981 Wave speed in noncircular collapsible ducts. Trans. ASME K: J. Biomech. Engng 103, 27-31.

Brower, R. W. \& Scholten, C. 1975 Experimental evidence on the mechanism for the instability of flow in collapsible vessels. Med. Biol. Engng 13, 839-845.

Canceldi, C. \& Pedley, T. J. 1985 A separated-flow model for collapsible-tube oscillations. J. Fluid Mech. 157, 375-404.

Conrad, W. A. 1969 Pressure-flow relationships in collapsible tubes. IEEE Trans. Bio-med. Engng $B M E-16,284-295$.

MoClurken, M. E., Kececioglu, I., Kamm, R. D. \& Shapiro, A. H. 1981 Steady, supercritical flow in collapsible tubes. Part 2. Theoretical studies. J. Fluid Mech. 109, 391-415.

OAtes, G. C. 1975 Fluid flow in soft-walled tubes. Part 1: Steady flow. Med. Biol. Engng 13, $773-784$.

REYN, J. W. 1987 Multiple solutions and flow limitation for steady flow through a collapsible tube held open at the ends. $J$. Fluid Mech. 174, 467-493.

Shapiro, A. H. 1977 a Physiologic and medical aspects of flow in collapsible tubes. Proc. 6th Canadian Congr. Appl. Mech., pp. 883-906.

ShapIRo, A. H. $1977 b$ Steady flow in collapsible tubes Trans. ASME K: J. Biomech. Engng 99, 126-147.

Sмiтн, F. T. \& DUCK, P. W. 1980 On the severe non-symmetric constriction, curving or cornering of channel flows. J. Fluid Mech. 98, 727-753. 